\title{
High-Pressure Processing vs. Thermal Treatment: Effect on the Stability of Polyphenols in Strawberry and Apple Products
}

\author{
Gabriela Lorena Salazar-Orbea ${ }^{1}$ (D), Rocío García-Villalba ${ }^{1}$, Francisco A. Tomás-Barberán ${ }^{1, *(D)}$ \\ and Luis Manuel Sánchez-Siles 2,3
}

1 Quality, Safety and Bioactivity of Plant-Derived Foods, Centro de Edafología y Biología Aplicada del Segura-Consejo Superior de Investigaciones Científicas (CEBAS-CSIC), 30100 Murcia, Spain; glsalazar@cebas.csic.es (G.L.S.-O.); rgvillalba@cebas.csic.es (R.G.-V.)

2 Research and Nutrition Department, Hero Group, 30820 Alcantarilla, Spain; luisma.sanchez@hero.es

3 Institute for Research and Nutrition, Hero Group, 5600 Lenzburg, Switzerland

* Correspondence: fatomas@cebas.csic.es

Citation: Salazar-Orbea, G.L.; García-Villalba, R.; Tomás-Barberán, F.A.; Sánchez-Siles, L.M. High-Pressure Processing vs. Thermal Treatment: Effect on the Stability of Polyphenols in Strawberry and Apple Products. Foods 2021, 10, 2919. https://doi.org/ $10.3390 /$ foods 10122919

\section{Academic Editors}

Francisco Artés-Hernández and Marina Cano Lamadrid

Received: 18 October 2021

Accepted: 22 November 2021

Published: 25 November 2021

Publisher's Note: MDPI stays neutra with regard to jurisdictional claims in published maps and institutional affiliations.

Copyright: (C) 2021 by the authors. Licensee MDPI, Basel, Switzerland. This article is an open access article distributed under the terms and conditions of the Creative Commons Attribution (CC BY) license (https:// creativecommons.org/licenses/by/ $4.0 /)$.

\begin{abstract}
Polyphenols are important bioactive compounds that are affected by processing. The consumer's demand for minimally processed products contributes to the increase in non-thermal technologies such as high-pressure processing (HPP) in the food industry. This review is aimed at critically discussing the positive and negative effects of thermal treatment (TT) and HPP on the stability of different polyphenol families in agro-food products obtained from strawberry and apple, two of the most used fruits in food processing. Our findings show that the phenolic content was affected by processing, fruit type, polyphenol family, and storage conditions (time and temperature) of the final product. To increase shelf life, manufacturers aiming to preserve the natural content of polyphenols need to find the sweet spot between polyphenol stability and product shelf-life since the residual enzyme activity from HPP can affect polyphenols negatively.
\end{abstract}

Keywords: polyphenols; apple; strawberry; bioactive compounds; food processing; high-pressure processing; thermal processing; stability; storage

\section{Introduction}

Strawberries and apples are fruits consumed worldwide, both fresh and processed (juices, jams, purees, smoothies, dried), due to their desirable sensory characteristics, nutritional value, and bioactive compounds. The main bioactive compounds found are polyphenols, the most significant dietary antioxidants present in fruit and vegetables, with a wide variety of biological activities linked to the associated health benefits [1]. These include flavonoid (flavonols, flavan-3-ols and proanthocyanidins, anthocyanins, dihydrochalcones, among others) and non-flavonoid (ellagitannins, ellagic acid, hydroxycinnamic acid derivatives, gallotannins) compounds. They are responsible for some of their organoleptic properties (color, aroma, astringency, bitterness) and also have relevant effects on nutrition and human health (antioxidants, anti-inflammatory, modulators of gut microbiota, etc.) [2]. There is considerable variability among the phenolic compounds found in strawberry and apple products (Figure 1), which depends on the inherent fruit characteristics (e.g., origin, variety, ripeness degree) as well as the type of food processing, and the part of the fruit (e.g., flesh, peel, achenes) [3,4].

In particular, in strawberries, flesh and achenes have a different polyphenol profile. In the flesh, the major phenols are flavan-3-ols/proanthocyanidins (F3OLs/PACs) and anthocyanins (ATs), while in achenes, the highest concentrations are for ellagitannins (ETs) and ellagic acid (EA) $[3,5,6]$. Similarly, in apples, there are differences in the phenolic compounds derived from flesh, peel, and seeds. In apple flesh, the major phenols are 
F3OLs/PACs and hydroxycinnamic acids (HCAs), being the latter mainly represented by chlorogenic acid. The principal polyphenols in apple peel are F3OLs/PACs having even higher concentrations than flesh, followed by flavonols (FOLs). In contrast, apple seeds are a more significant source of dihydrochalcones (DHCs) $[4,7,8]$.

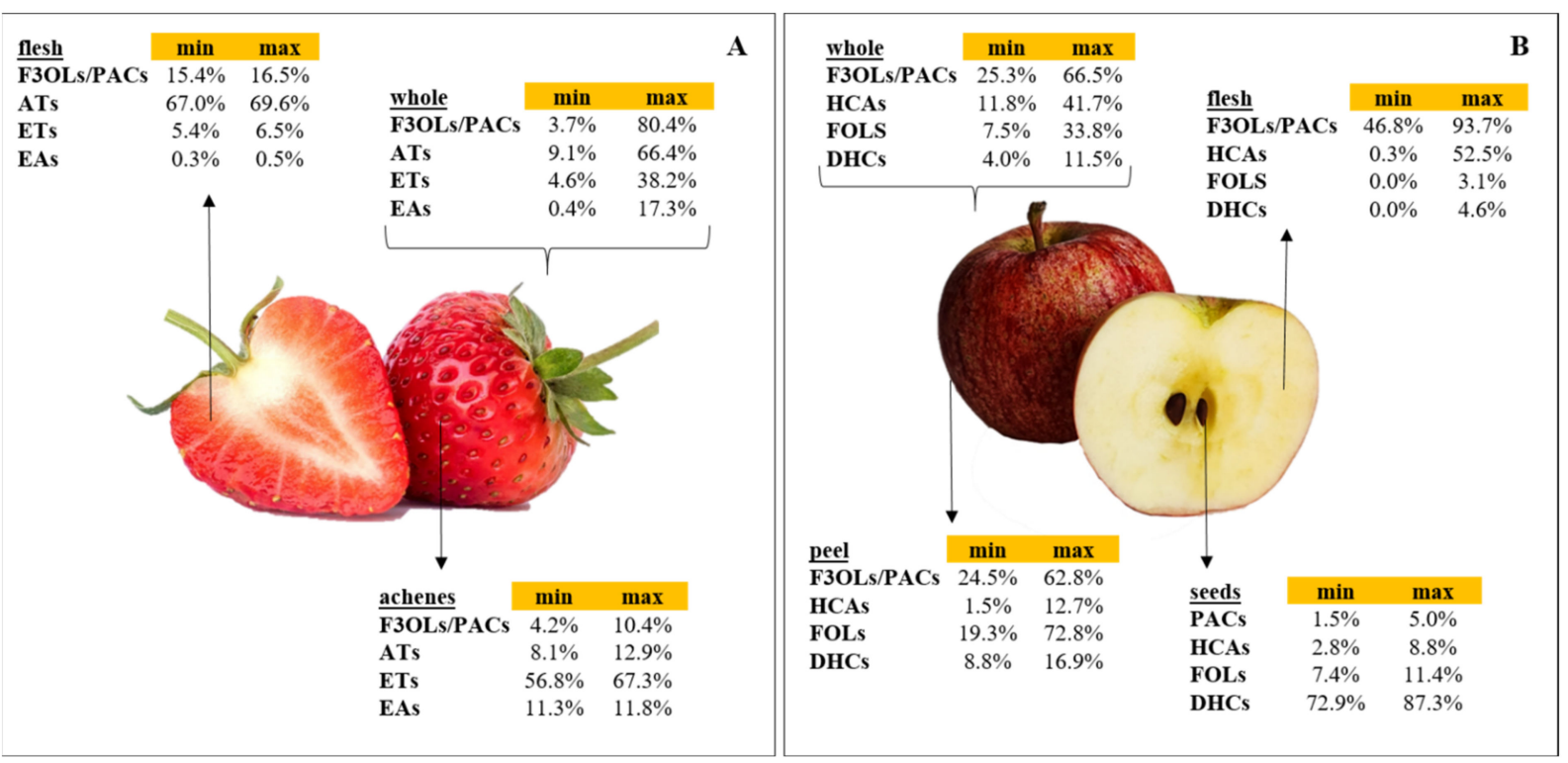

Figure 1. Percentage contribution of the main phenol families to the total polyphenols in different tissues of strawberry (A) and apple (B). (F3OLs/PACs) flavan-3-ols/proanthocyanidins, (ATs) anthocyanins, (ETs) ellagitannins, (EAs) ellagic acid and conjugates, (HCAs) hydroxycinnamic acids, (FOLs) flavonols, (DHCs) dihydrochalcones, (PACs) proanthocyanidins. Data retrieved from [3-6,8-13].

There is growing consumer awareness of the importance of consuming food products that preserve nutritional and functional properties [14]. Consumers' concern for food processing is increasing in many dimensions: from its link to healthiness and naturalness to its impact on the environment [15]. Nevertheless, preservation techniques are required to retain the nutritional value, ensure food safety during shelf life, and maintain its organoleptic characteristics. A wide variety of industrial thermal and non-thermal treatment technologies are available for food preservation. Thermal treatments include conventional techniques such as pasteurization and sterilization and new thermal technologies such as ohmic heating and dielectric heating (radiofrequency and microwave).

Meanwhile, non-thermal treatments include high-pressure processing, dehydration, freezing, pulsed electric fields, cold plasma, ultrasound processing, magnetic field, and ozone $[16,17]$. Thermal treatment is the most widely used preservation technique. According to the intensity of heat treatment, thermal treatments may be differentiated into pasteurization $\left(70-80^{\circ} \mathrm{C}\right)$, sterilization $\left(110-120^{\circ} \mathrm{C}\right)$, and ultra-high-temperature treatment $\left(140-160{ }^{\circ} \mathrm{C}\right)$ [18]. Although thermal treatments produce some adverse effects, such as loss of some nutrients (vitamin C), formation of undesirable compounds (acrylamide, heterocyclic amines, sulfur compounds), and in some cases the degradation of organoleptic characteristics, it also exerts many beneficial effects such as inactivation of foodborne pathogens, inactivation of toxins and enzymes polyphenoloxidase (PPO), peroxidase (POD), pectin-methyl esterase, improvement of digestibility and bioavailability of nutrients, improvement of some sensory characteristics (texture and flavor), enhancement of extraction of health beneficial compounds and extension of shelf life $[19,20]$. Strawberry and apple polyphenol oxidases and peroxidases are responsible for the oxidation of phenolic compounds when fruit tissues are disrupted in the presence of oxygen, leading to the formation of brown polymers and other discolorations that affect the organoleptic properties of the fruit and derived products negatively. 
Furthermore, one of the most studied non-thermal treatments is HPP. It works with high hydrostatic pressure and is commonly used at the industrial level between 300 and $600 \mathrm{MPa}$ for 3-5 min [21,22]. As a consequence of pressure, there may be some changes in physical properties (solubility, density, viscosity), kinetic reactions (acceleration or delay of reactions rate), as well as, some equilibrium processes (dissociation of weak acids, acid-base equilibria, and ionization) [23]. The effect of pressure on food components depends on the type of bond between their molecules and the interatomic distance. In general, the high-pressure treatment minimally affects compounds with covalent bonds (vitamins, minerals, folates, antioxidants, anthocyanins, and flavor compounds) [17,24]. A disadvantage of HPP is that it is not entirely effective in polyphenol oxidase, peroxidase, and pectin methylesterase inactivation at the industrial processing conditions and subsequent storage [25]. Therefore, it triggers oxidation reactions that degrade phenolic compounds and shorten the shelf life $[26,27]$.

Briefly, given the above facts and considerations summarized as follows: (1) polyphenols are important bioactive compounds that are affected by processing, (2) consumers are increasingly demanding "minimally processed" products, and (3) several non-thermal processing technologies such as HPP are now prevalent on the market, here, the objective of this article is to conduct a review of the literature about the differences between TT and HPP on the impact in polyphenol degradation in strawberry and apple products, two of the most used fruits in food processing.

\section{Overview of the Studies Included in the Review}

We systematically searched, evaluated, and synthesized research articles on the effects of traditional TT and HPP and their subsequent storage on the main polyphenols found in strawberries and apples. In strawberries, we analyzed F3OLs/PACs, ATs, ETs, and EA. Whereas for apple, F3OLs/PACs, FOLs, DHCs, and HCAs were reviewed. The major databases for the topic were used (Web of Science, ScienceDirect, SCOPUS, PubMed, and Google Scholar). The search terms used were: polyphenols or proanthocyanidins or anthocyanins or ellagic acid or flavonols or dihydrochalcones or hydroxycinnamic acids and thermal treatment or high-pressure processing or high hydrostatic pressure and strawberry or apple.

The search was focused on studies published over the last 10 years. Articles were included: (i) if the phenols were quantified by high-performance liquid chromatography (HPLC); (ii) when at least three studies were found for strawberry and apple identifying the effect of the related processing technology; (iii) when there was a control of fresh strawberry and apple or their unprocessed products. Articles were excluded: (i) when the analyzed sample was a mix of more than one fruit; (ii) when the HPP processing was combined with any thermal treatment higher than $50{ }^{\circ} \mathrm{C}$; (iii) when the TT led to a concentration or reduction in the final product, e.g., jams.

For comparison purposes, the quantification of the different strawberry and apple polyphenols in the literature were converted to $\mathrm{mg} / 100 \mathrm{~g} \mathrm{FW}$ (fresh weight) to provide values closer to the fruit servings used in nutritional studies. The conversion of values based on DW (dry weight) to FW assumed that a fruit retains 10\% of the original fresh weight after drying.

Overall, 26 articles were reviewed to address the study question: what are the effects of TT and HPP on the stability of polyphenols?

- Strawberry was the most studied fruit with 15 articles, whereas 11 were examined for apple products;

- A total of 19 articles reported the effects of thermal treatment and 12 for high-pressure processing. The reviewed studies examined 199 different trials at different processing and storage conditions (Figure 2). 

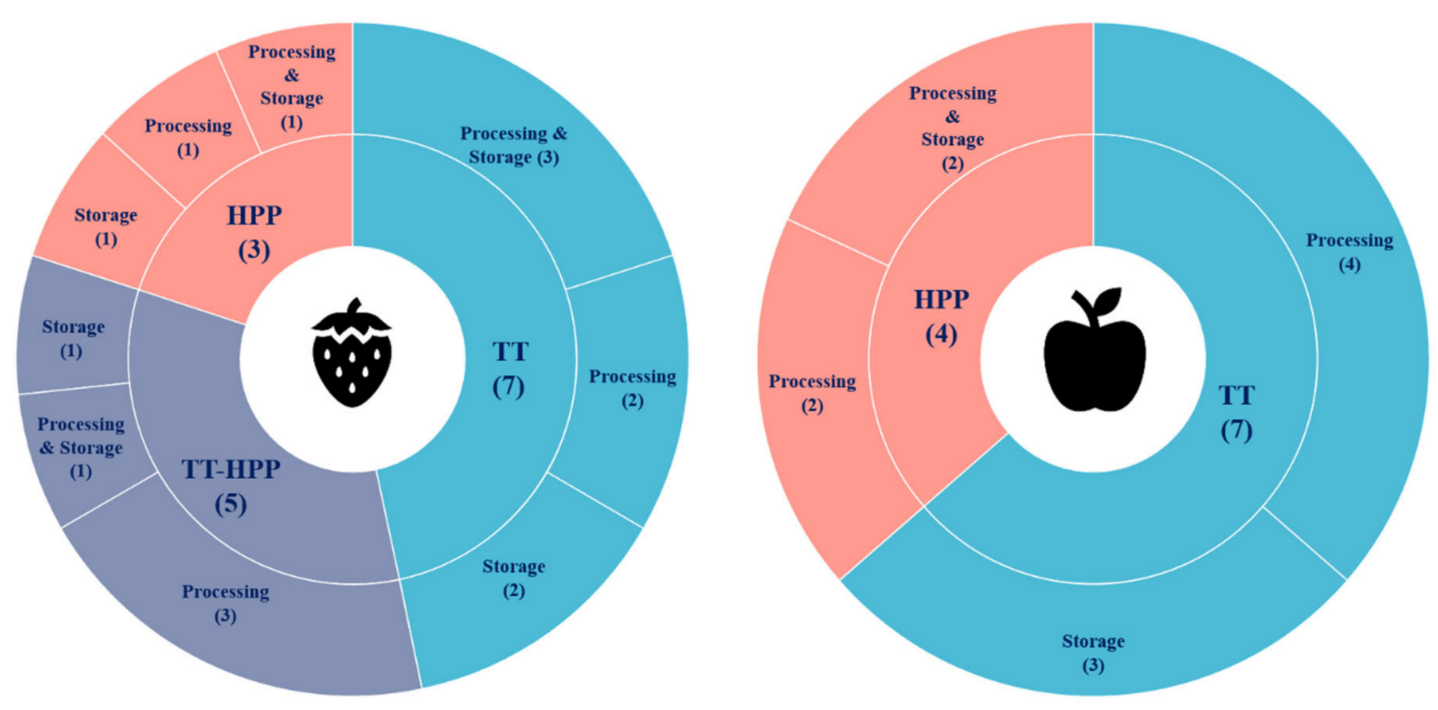

Thermal Treatment (19 studies)

High Pressure Processing (12 studies)

Figure 2. Overview of the studies evaluating the thermal treatment (TT) and high-pressure processing (HPP) impact and their subsequent storage on the primary polyphenols in strawberry and apple products.

The concentrations reported in these studies for phenols of the same family were added. Each group's total polyphenols were compared before and after processing, obtaining a percentage of increase or decrease attributed to the processing effect. A total of 4 of the 26 articles reviewed did not provide quantitative information to calculate these percentages, and therefore they were not included in the figures. The phenolic concentrations recorded after processing were considered control to determine the storage effect, and the results obtained during storage were compared against the control.

\section{Effects of Processing and Storage Conditions on the Stability of Polyphenols}

This section will discuss the positive and negative effects of TT and HPP treatments and their subsequent storage on the stability of the main polyphenols in strawberries (Table 1) and apples (Table 2). A summary is presented in Table 3.

\subsection{Effects on the Stability of Proanthocyanidins in Strawberry and Apple Products}

Proanthocyanidins, or condensed tannins, are plant secondary metabolites that could be found as oligomers or polymers of flavan-3-ol monomeric units. The most common monomeric building blocks are catechin, (epi)-catechin, (epi)-afzelechin, and (epi)gallocatechin [28]. F3OLs/PACs are found in a wide variety of fruits, nuts, legumes, and cereals. The ranges reported highly depend on whether the study measured flavan-3-ol monomers (F3OLs), oligomers, and polymers or flavanol-3-ol units after hydrolysis (PACs). For most of the studies published, F3OLs and PACs were not differentiated, and therefore here we consider them together (F3OLs/PACs) unless differentiation is made.

In the case of strawberry, Nowicka et al. [3] recorded ranges of PACs polymers between 3.4 and $37.5 \mathrm{mg} / 100 \mathrm{~g}$ FW, while Buendia et al. [6] registered levels of total flavan-3-ol monomers from 53.9 to $168.1 \mathrm{mg} / 100 \mathrm{~g}$ FW after phloroglucinolysis. For apple, studies reported PACs polymers concentration from 12.6 to $203.3 \mathrm{mg} / 100 \mathrm{~g}$ FW in the whole fruit $[12,29]$. Nevertheless, higher levels were quantified for F3OLs units in peel and flesh. The flesh has from 1.4 to $548.2 \mathrm{mg} / 100 \mathrm{~g} \mathrm{FW}$, whereas in the peel, levels varied between 41.4 and $171.2 \mathrm{mg} / 100 \mathrm{~g}$ FW [7]. Numerous health benefits have been attributed to F3OLs/PACs, such as free radical scavenging, antioxidant, antiviral, antibacterial, anticarcinogenic, anti-inflammatory, and anti-allergic activities [28,30]. 
Table 1. Effects of TT and HPP treatments and storage on the main phenolic compounds in strawberry products.

\begin{tabular}{|c|c|c|c|c|c|c|}
\hline Material & Treatment: Conditions & Storage & $\begin{array}{l}\text { Impact of Processing Conditions } \\
\text { on Polyphenols }\end{array}$ & $\begin{array}{c}\text { Impact of Storage } \\
\text { Conditions on Polyphenols }\end{array}$ & Mechanisms & Ref. \\
\hline $\begin{array}{l}\text { Strawberry } \\
\text { fresh }\end{array}$ & $\begin{array}{l}\text { TT: } \\
90^{\circ} \mathrm{C} / 5 \mathrm{~min}\end{array}$ & $\begin{array}{l}360 \text { days } \\
-20^{\circ} \mathrm{C}\end{array}$ & $\begin{array}{l}\text { F3OLs } / \text { PACs: } \uparrow 30 \% \text { CA, } \uparrow 73 \% \text { EGC; } \\
\uparrow 45 \% \text { EC } \\
\text { ATs: } \downarrow 16 \% \text { pel-3-glu; } \downarrow 5 \% \text { pel-3-rut; } \\
\approx \text { cya-3-glu } \\
\text { EA: } \uparrow 143 \%\end{array}$ & $\begin{array}{l}\text { F3OLs/PACs: } \downarrow 19 \% \text { CA, } \downarrow 39 \% \\
\text { EGC } \\
\text { ATs: } \downarrow \text { pel-3-glu; } \downarrow \text { pel-3-rut; } \\
\uparrow \text { cya-3-glu } \\
\text { EA: } \downarrow 65 \%\end{array}$ & $\begin{array}{l}\uparrow F 3 O L s / \text { PACs: cleavage } \rightarrow \text { release of } \\
\text { dimers and monomers; release from } \\
\text { cellular tissue } \\
\downarrow \text { ATs: cleavage of covalent bonds, } \\
\text { polymerization, and derivatization; } \uparrow p H \text { in } \\
\text { the food matrix; enzymatic oxidation after } \\
\text { pasteurization and during storage } \\
\uparrow E A: \text { ETs hydrolysis }\end{array}$ & {$[31]^{\mathrm{d}}$} \\
\hline $\begin{array}{l}\text { Strawberry } \\
\text { fresh }\end{array}$ & $\begin{array}{l}\mathrm{TT}: \\
90^{\circ} \mathrm{C} / 5 \mathrm{~min}\end{array}$ & $\begin{array}{l}90 \text { days } \\
23^{\circ} \mathrm{C}\end{array}$ & $\begin{array}{l}\text { F3OLs/PACs: } \uparrow 34 \% \text { CA; } \uparrow 134 \% \text { EC; } \\
\uparrow 119 \% \text { EGCG; } \uparrow 30 \% \text { T.F3OLs/PACs } \\
\text { ATs: } \downarrow 30 \% \text { cya-3-glu; } \downarrow 35 \% \\
\text { pel-3-glu; } \approx \text { pel-3-rut; } \downarrow 30 \% \text { T.ATs } \\
\text { EA: } \uparrow 66 \%\end{array}$ & $\begin{array}{l}\text { F3OLs } / \text { PACs: } \downarrow 42 \% \text { CA; } \downarrow 62 \% \\
\text { EGCG; } \downarrow 67 \% \text { EC } \\
\text { ATs: } \downarrow 87 \% \text { cya-3-glu, } \downarrow 97 \% \\
\text { pel-3-glu; } \downarrow 92 \% \text { pel-3-rut } \\
\text { EA: } \downarrow 32 \%\end{array}$ & $\begin{array}{l}\text { TT } \\
\uparrow \text { F3OLs/PACs: PACs cleavage } \rightarrow \text { release of } \\
\text { dimers and monomers; release from } \\
\text { cellular tissue } \\
\downarrow \text { ATs: cleavage of covalent bonds, } \\
\text { polymerization, and derivatization } \\
\uparrow \text { EA: release from cell walls; hydrolysis } \\
\text { from ETs to EA } \\
\text { Storage } \\
\downarrow \text { F3OLs } / \text { PACs, } \downarrow \text { ATs and } \downarrow \text { EA: oxidation; } \\
\text { non-ezymatic and enzymatic oxidation }\end{array}$ & [32] \\
\hline $\begin{array}{l}\text { Strawberry } \\
\text { puree }\end{array}$ & $\begin{array}{l}\text { TT1 (SB): } \\
85^{\circ} \mathrm{C} / 3 \text { min } \\
\text { TT2 (P): } \\
85^{\circ} \mathrm{C} / 3 \text { min }\end{array}$ & No & $\begin{array}{l}\text { SB: } \\
\text { F3OLs/PACs: from } \uparrow 42 \% \text { to } \downarrow 16 \% \\
\text { ATs: } \downarrow 34-43 \% \\
\text { P: } \\
\text { F3OLs/PACs: from } \uparrow 53 \% \text { to } \downarrow 35 \% \\
\text { ATs: } \uparrow 2-18 \%\end{array}$ & - & $\begin{array}{l}\downarrow \text { F3OLs/PACs: TT conditions degraded } \\
\text { heat-labile flavan-3-ols } \\
\downarrow \text { ATs: SB may influence the ATs stability }\end{array}$ & [33] \\
\hline $\begin{array}{l}\text { Strawberry } \\
\text { puree } \\
\text { ( } 2 \text { yearsharvest })\end{array}$ & $\begin{array}{l}\text { TT: } \\
90^{\circ} \mathrm{C} / 2 \mathrm{~min}\end{array}$ & No & $\begin{array}{l}\text { Puree with seeds } \\
\text { EA: } \downarrow 8 \% \text { (2011 harvest), } \\
\downarrow 13 \% \text { (2012 harvest) } \\
\text { Puree without seeds } \\
\text { EA: } \downarrow 35 \% \text { (2011 harvest) } \\
\downarrow 19 \% \text { EA (2012 harvest) }\end{array}$ & - & $\downarrow$ EA: oxidation by membrane breakage & [34] \\
\hline
\end{tabular}


Table 1. Cont

\begin{tabular}{|c|c|c|c|c|c|c|}
\hline Material & Treatment: Conditions & Storage & $\begin{array}{l}\text { Impact of Processing Conditions } \\
\text { on Polyphenols a }\end{array}$ & $\begin{array}{l}\text { Impact of Storage } \\
\text { Conditions on Polyphenols }\end{array}$ & Mechanisms & Ref. \\
\hline $\begin{array}{l}\text { Strawberry } \\
\text { puree }\end{array}$ & $\begin{array}{l}\text { TT: } \\
100{ }^{\circ} \mathrm{C} / 10 \mathrm{~min}\end{array}$ & $\begin{array}{l}8 \text { weeks } \\
25^{\circ} \mathrm{C}\end{array}$ & - & $\begin{array}{l}\text { ATs: } \downarrow \text { } \downarrow 0-88 \% \text { pel-3-glu (8 } \\
\text { weeks); } \downarrow 53-74 \% \text { pel-3-rut (2 } \\
\text { weeks); } \downarrow 63-78 \% \\
\text { pel-3-mal-glu ( } 2 \text { weeks); } \\
\downarrow 70-86 \% \text { pel-3-ace-glu (2 } \\
\text { weeks); } \downarrow 90-93 \% \text { T.ATs (8 } \\
\text { weeks) }\end{array}$ & $\begin{array}{l}\downarrow \text { ATs: oxidation by PPO; formation of dark } \\
\text { condensation products }\end{array}$ & [35] \\
\hline $\begin{array}{l}\text { Strawberry } \\
\text { puree } \\
\text { ( } 2 \text { varieties) }\end{array}$ & $\begin{array}{l}\text { TT1 (SB): } \\
100^{\circ} \mathrm{C} / 3 \mathrm{~min} \\
\text { TT2 (P): } \\
60,75,90^{\circ} \mathrm{C} / 3 \mathrm{~min}\end{array}$ & $\begin{array}{l}28 \text { days } \\
20^{\circ} \mathrm{C}\end{array}$ & $\begin{array}{l}\text { Ats-Elsanta var.: from } \approx \text { to } \downarrow 5 \% \\
(P 60 ; \text { P75); } \downarrow 10-12 \%(S B ; P 90) \\
\text { ATs-Everest var.: } \approx(P 90) ; \downarrow 8 \%(S B, \\
P 60, P 75)\end{array}$ & $\begin{array}{l}\text { Elsanta var.: } \downarrow 62-65 \% \text { T.ATs } \\
\text { (all treatments) } \\
\text { Everest var.: } \downarrow 64-73 \% \text { T.ATs } \\
\text { (all treatments) }\end{array}$ & $\begin{array}{l}\text { SB and P } \\
\downarrow \text { ATs: cleavage of covalent bonds, } \\
\text { polymerization, and derivatization } \\
\text { Storage } \\
\downarrow \text { ATs: PPO partial reactivation during } \\
\text { storage } \rightarrow \text { oxidation }\end{array}$ & [36] \\
\hline $\begin{array}{l}\text { Strawberry } \\
\text { juice } \\
\text { (7 varieties) }\end{array}$ & $\begin{array}{l}\text { TT: } \\
90^{\circ} \mathrm{C} / 2 \mathrm{~min}\end{array}$ & $\begin{array}{l}6 \text { months at } 4 \\
\text { and } 20^{\circ} \mathrm{C}\end{array}$ & - & $\begin{array}{l}4{ }^{\circ} \mathrm{C}: \\
\text { F3OLs/PACs: from } \downarrow 0.3 \% \text { to } \\
\uparrow 27 \% \text { polymeric PACs } \\
\text { ATs: } \downarrow 69 \% \text { pel-3-glu; } \downarrow 73 \% \\
\text { pel-3-mal-lglu; } \downarrow 68 \% \\
\text { cya-3-glu; } \downarrow 56 \% \text { cya-3-mal-glu; } \\
\downarrow 59-89 \% \text { T.ATs } \\
20{ }^{\circ} \mathrm{C} \text { : } \\
\text { F3OLs/PACs: from } \downarrow 10 \% \text { to } \\
\uparrow 11 \% \text { polymeric PACs } \\
\text { ATs: } \downarrow 97 \% \text { pel-3-glu; } \downarrow 99 \% \\
\text { pel-3-malonylglu; } \downarrow 98 \% \\
\text { cya-3-glu; } \downarrow 85 \% \text { cya-3-mal-glu; } \\
\downarrow 94-99 \% \text { T.ATs }\end{array}$ & $\begin{array}{l}\uparrow \text { F3OLs/PACs: protective effect of colloidal } \\
\text { suspensions } \\
\downarrow \text { F3OLs/PACs and } \downarrow \text { ATs: cleavage of } \\
\text { covalent bonds, polymerization, and } \\
\text { derivatization; enzymatic oxidation after } \\
\text { pasteurization and during storage }\end{array}$ & [37] \\
\hline $\begin{array}{l}\text { Strawberry } \\
\text { puree }\end{array}$ & $\begin{array}{l}\mathrm{HPP}: 100-400 \mathrm{MPa} \\
/ 15 \mathrm{~min} \text { at } 20 \text { and } 50{ }^{\circ} \mathrm{C}\end{array}$ & No & $\begin{array}{l}\text { ATs: } \approx \text { pel-3-glu; } \approx \text { cya-3-glu; } \\
\approx \text { pel-3-rut }(\text { all HPP); from } \approx \text { to } \\
\uparrow 15 \% \text { T.ATs }\end{array}$ & - & $\approx$ ATs: Sufficient enzyme inactivation & [38] \\
\hline
\end{tabular}


Table 1. Cont

\begin{tabular}{|c|c|c|c|c|c|c|}
\hline Material & Treatment: Conditions & Storage & $\begin{array}{l}\text { Impact of Processing Conditions } \\
\text { on Polyphenols }\end{array}$ & $\begin{array}{c}\text { Impact of Storage } \\
\text { Conditions on Polyphenols } b\end{array}$ & Mechanisms & Ref. \\
\hline $\begin{array}{l}\text { Strawberry } \\
\text { puree } \\
\text { (2 years harvest) }\end{array}$ & $\begin{array}{l}\text { HPP: } \\
300 \text { and } 600 \mathrm{MPa} / \\
15 \text { min at } 50^{\circ} \mathrm{C}\end{array}$ & $\begin{array}{l}28 \text { weeks } \\
6{ }^{\circ} \mathrm{C}\end{array}$ & $\begin{array}{l}\text { ATs - 300 MPa harvest 2011: } \downarrow 12 \% \\
\text { cy-3-glu; } \downarrow 13 \% \text { pel-3-glu; } \downarrow 36 \% \\
\text { pel-3-rut; } \downarrow 15 \% \text { T.ATs } \\
\text { ATs - 600 MPa harvest 2012: } \downarrow 22 \% \\
\text { cy-3-glu; } \downarrow 21 \% \text { pel-3-glu; } \downarrow 10 \% \\
\text { pel-3-rut; } \downarrow 21 \% \text { T.ATs }\end{array}$ & $\begin{array}{l}\text { ATs-300 MPa harvest 2011: } 86 \\
\text { days half-life } \\
\text { ATs-600 MPa harvest 2012: } 62 \\
\text { days half-life }\end{array}$ & $\downarrow$ ATs: residual PPO activity $\rightarrow$ oxidation & [39] \\
\hline $\begin{array}{l}\text { Strawberry } \\
\text { pure }\end{array}$ & $\begin{array}{l}\text { TT: } \\
72^{\circ} \mathrm{C} / 1 \mathrm{~min} \\
\mathrm{HPP}: \\
600 \mathrm{MPa} / 1 \mathrm{~min}\end{array}$ & No & $\begin{array}{l}\text { TT } \\
\text { F3OLs/PACs: } \uparrow 122 \% \text { CA; } \uparrow 33 \% \\
\text { proanthocyanidin } \mathrm{B} 1 ; \uparrow 78 \% \\
\text { T.F3OLs/PACs } \\
\text { ATs: } \uparrow 40 \% \text { cya-3-O-glu; } \uparrow 26 \% \\
\text { pel-3-O-glu; } \uparrow 22 \% \text { pel-3-O-rut; } \uparrow 34 \% \\
\text { pel-3-O-mal-glu; } \uparrow 39 \% \\
\text { pel-3-O-acetylglu; } \uparrow 32 \% \text { T.ATs } \\
\text { EA: } \uparrow 8 \% \\
\text { HPP } \\
\text { F3OLs/PACs: } \uparrow 68 \% \text { CA; } \uparrow 19 \% \\
\text { proanthocyanidin B1; } \uparrow 43 \% \\
\text { T.F3OLs/PACs } \\
\text { ATs: } \uparrow 12 \% \text { cya-3-O-glu; } \uparrow 8 \% \\
\text { pel-3-O-glu; } \uparrow 10 \% \text { pel-3-O-rut; } \uparrow 13 \% \\
\text { pel-3-O-mal-glu; } \uparrow 12 \% \\
\text { pel-3-O-ace-glu; } \uparrow 11 \% \text { T.ATs } \\
\text { EA: } \approx\end{array}$ & - & $\begin{array}{l}\text { TT } \\
\uparrow F 3 O L s / P A C s: \text { cleavage } \rightarrow \text { release of } \\
\text { dimers and monomers } \\
\uparrow A T s: \text { higher extraction from cell matrix } \\
\text { favored by TT } \\
\text { HPP: } \\
\uparrow F 3 O L s / \text { PACs: release from the disrupted } \\
\text { cell walls }\end{array}$ & [40] \\
\hline
\end{tabular}


Table 1. Cont.

\begin{tabular}{|c|c|c|c|c|c|c|}
\hline Material & Treatment: Conditions & Storage & $\begin{array}{l}\text { Impact of Processing Conditions } \\
\text { on Polyphenols a }\end{array}$ & $\begin{array}{c}\text { Impact of Storage } \\
\text { Conditions on Polyphenols }\end{array}$ & Mechanisms & Ref. \\
\hline $\begin{array}{l}\text { Strawberry } \\
\text { puree }\end{array}$ & $\begin{array}{l}\text { TT: } \\
90{ }^{\circ} \mathrm{C} \text { for } 15 \mathrm{~min} \\
\mathrm{HPP} \text { : } \\
300 \text { and } 500 \mathrm{MPa} / \\
1,5,15 \mathrm{~min} \text { at } 0{ }^{\circ} \mathrm{C}\end{array}$ & No & $\begin{array}{l}\text { TT } \\
\text { ATs: } \downarrow 44 \% \text { cya-3-glu; } \downarrow 43 \% \\
\text { pel-3-glu; } \downarrow 49 \% \text { pel-3-rut; } \downarrow 44 \% \\
\text { T.ATs } \\
\text { EA: } \uparrow 30.5 \% \text { EA } \\
\text { HPP }-0{ }^{\circ} \mathrm{C} \text { (all HPP conditions) } \\
\text { ATs: } \downarrow 5 \% \text { cya-3-glu; } \downarrow 7 \% \text { pel-3-glu; } \\
\downarrow 15 \% \text { pel-3-rut; } \downarrow 7 \% \text { T. ATs } \\
\text { EA: } \approx \text { EA ( } 300 \text { and } 600 \mathrm{MPa}) \\
\text { HPP }+50{ }^{\circ} \mathrm{C} \\
\text { ATs: } \downarrow 14 \% \text { cya-3-glu; } \downarrow 13 \% \\
\text { pel-3-glu; } \downarrow 31 \% \text { pel-3-rut; } \downarrow 14 \% \\
\text { T.ATs } \\
\text { EA: } \uparrow 28.4 \% \text { (300 MPa); } \uparrow 15.5 \%(600 \\
\text { MPa) }\end{array}$ & - & $\begin{array}{l}\text { TT } \\
\downarrow \text { ATs: cleavage of covalent bonds, } \\
\text { polymerization, and derivatization } \\
\uparrow E A: \text { release from the achenes favored by } \\
\text { TT } \\
\text { HPP }-0^{\circ} \mathrm{C} \\
\downarrow \text { ATs: insufficient enzyme inactivation } \\
\text { (PPO and POD) } \rightarrow \text { oxidation } \\
\text { HPP }+50^{\circ} \mathrm{C} \\
\downarrow \text { ATs: formation of colorless chalcones } \\
\uparrow E A: \text { release from ETs }\end{array}$ & [41] \\
\hline
\end{tabular}


Table 1. Cont

\begin{tabular}{|c|c|c|c|c|c|c|}
\hline Material & Treatment: Conditions & Storage & $\begin{array}{c}\text { Impact of Processing Conditions } \\
\text { on Polyphenols }\end{array}$ & $\begin{array}{c}\text { Impact of Storage } \\
\text { Conditions on Polyphenols } b\end{array}$ & Mechanisms & Ref. \\
\hline $\begin{array}{l}\text { Strawberry } \\
\text { Puree } \\
\text { c (3 varieties) }\end{array}$ & $\begin{array}{l}\text { TT: } \\
88^{\circ} \mathrm{C} / 2 \mathrm{~min} \\
\mathrm{HPP}: \\
600 \mathrm{MPa} / 5 \mathrm{~min} \\
\text { at } 20^{\circ} \mathrm{C}\end{array}$ & $\begin{array}{l}3 \text { months } \\
4{ }^{\circ} \mathrm{C}\end{array}$ & $\begin{array}{l}\text { TT } \\
\text { Camarosa var. } \\
\text { ATs: } \downarrow 16 \% \text { cya-3-glu; } \downarrow 23 \% \\
\text { pel-3-glu; } \downarrow 26 \% \text { pel-3-rut; } \downarrow 22 \% \\
\text { T.ATs } \\
\text { Rubygem var. } \\
\text { ATs: } \downarrow 42 \% \text { cya-3-glu; } \downarrow 24 \% \\
\text { pel-3-glu; } \downarrow 29 \% \text { pel-3-rut; } \downarrow 25 \% \\
\text { T.ATs } \\
\text { Festival } \\
\text { ATs: } \downarrow 27 \% \text { cya-3-glu; } \downarrow 26 \% \\
\text { pel-3-glu; } \downarrow 26 \% \text { pel-3-rut; } \downarrow 26 \% \\
\text { T.ATs } \\
\text { HPP } \\
\text { Camarosa var. } \\
\text { ATs: } \downarrow 22 \% \text { cya-3-glu; } \downarrow 26 \% \\
\text { pel-3-glu; } \downarrow 28 \% \text { pel-3-rut; } \downarrow 26 \% \\
\text { T.ATs } \\
\text { Rubygem var. } \\
\text { ATs: } \downarrow 42 \% \text { cya-3-glu; } \downarrow 27 \% \\
\text { pel-3-glu; } \downarrow 32 \% \text { pel-3-rut; } \downarrow 28 \% \\
\text { T.ATs } \\
\text { Festival } \\
\text { ATs: } \downarrow 17 \% \text { cya-3-glu; } \downarrow 20 \% \\
\text { pel-3-glu; } \downarrow 18 \% \text { pel-3-rut; } \downarrow 20 \% \\
\text { T.ATs }\end{array}$ & $\begin{array}{l}\text { TT } \\
\text { Camarosa var. } \\
\text { ATs: } \downarrow 66 \% \text { cya-3-glu; } \downarrow 69 \% \\
\text { pel-3-glu; } \downarrow 60 \% \text { pel-3-rut; } \\
\downarrow 68 \% \text { T.ATs } \\
\text { Rubygem var. } \\
\text { ATs: } \downarrow 52 \% \text { cya-3-glu; } \downarrow 69 \% \\
\text { pel-3-glu; } \downarrow 59 \% \text { pel-3-rut; } \\
\downarrow 68 \% \text { T.ATs } \\
\text { Festival } \\
\text { ATs: } \downarrow 59 \% \text { cya-3-glu; } \downarrow 65 \% \\
\text { pel-3-glu; } \downarrow 59 \% \text { pel-3-rut; } \\
\downarrow 65 \% \text { T.ATs } \\
\text { HPP } \\
\text { Camarosa var. } \\
\text { ATs: } \downarrow 69 \% \text { cya-3-glu; } \downarrow 72 \% \\
\text { pel-3-glu; } \downarrow 69 \% \text { pel-3-rut; } \\
\downarrow 72 \% \text { T.ATs } \\
\text { Rubygem var. } \\
\text { ATs: } \downarrow 62 \% \text { cya-3-glu; } \downarrow 75 \% \\
\text { pel-3-glu; } \downarrow 71 \% \text { pel-3-rut; } \\
\downarrow 75 \% \text { T.ATs } \\
\text { Festival } \\
\text { ATs: } \downarrow 73 \% \text { cya-3-glu; } \downarrow 77 \% \\
\text { pel-3-glu; } \downarrow 76 \% \text { pel-3-rut; } \\
\downarrow 76 \% \text { T.ATs }\end{array}$ & $\begin{array}{l}\downarrow \text { ATs: partially due to variety effect } \\
\text { PPO and POD; oxidation and co-oxidation; } \\
\text { non-enzymatic reactions; } \\
\text { cleavage of covalent bonds }\end{array}$ & [42] \\
\hline
\end{tabular}


Table 1. Cont

\begin{tabular}{|c|c|c|c|c|c|c|}
\hline Material & Treatment: Conditions & Storage & $\begin{array}{l}\text { Impact of Processing Conditions } \\
\text { on Polyphenols }\end{array}$ & $\begin{array}{l}\text { Impact of Storage } \\
\text { Conditions on Polyphenols } b\end{array}$ & Mechanisms & Ref. \\
\hline $\begin{array}{l}\text { Strawberry } \\
\text { pure }\end{array}$ & $\begin{array}{l}\text { TT: } \\
90{ }^{\circ} \mathrm{C} \text { for } 15 \mathrm{~min} \\
\mathrm{HPP}: \\
500 \mathrm{MPa} / 15 \mathrm{~min} \\
\text { at } 50^{\circ} \mathrm{C}\end{array}$ & $\begin{array}{l}12 \text { weeks } \\
6{ }^{\circ} \mathrm{C}\end{array}$ & - & $\begin{array}{l}\text { TT } \\
\text { ATs: } \downarrow 17 \% \text { cya-3-glu; } \downarrow 19 \% \\
\text { pel-3-glu; } \downarrow 19 \% \text { pel-3-rut; } \\
\downarrow 19 \% \text { T.ATs } \\
\text { EA: } \uparrow 56 \% \text { EA until week } 10 \\
\text { HPP }+50{ }^{\circ} \mathrm{C} \\
\text { ATs: } \downarrow 72 \% \text { cya-3-glu; } \downarrow 68 \% \\
\text { pel-3-glu; } \downarrow 72 \% \text { pel-3-rut; } \\
\downarrow 69 \% \text { T.ATs } \\
\text { EA: } \uparrow 43 \% \text { EA until the end of } \\
\text { storage }\end{array}$ & $\begin{array}{l}\text { TT-Storage } \\
\uparrow E A: \text { release from the achenes; ETs } \\
\text { hydrolysis; low pH increased ETs } \\
\text { hydrolysis } \\
\text { HPP Storage } \\
\downarrow \text { ATs: not enough enzyme inactivation PPO } \\
\text { and POD } \rightarrow \text { oxidation }\end{array}$ & [43] \\
\hline $\begin{array}{l}\text { Strawberry } \\
\text { pulp }\end{array}$ & $\begin{array}{l}\mathrm{TT}: \\
70^{\circ} \mathrm{C} / 2 \mathrm{~min} \\
\mathrm{HPP}: \\
400,500,600 \mathrm{MPa} / \\
5,10,15,20,25 \mathrm{~min} \\
\text { at } 25^{\circ} \mathrm{C}\end{array}$ & No & $\begin{array}{l}\text { TT } \\
\text { F3OLs/PACs: } \uparrow 42 \% \text { CA } \\
\text { ATs: } \downarrow 17 \% \text { cy-3-glu, } \downarrow 23 \% \text { pel-3-glu, } \\
\downarrow 21 \% \text { pel-3-rut; } \downarrow 22 \% \text { T.ATs } \\
\text { EA: } \uparrow 17 \% \\
\text { HPP } \\
\text { F3OLs/PACs: } \approx \text { CA ( } 500 \mathrm{MPa} / 20, \\
25 \mathrm{~min} ; 600 \mathrm{MPa} / 5-25 \mathrm{~min}) ; \downarrow 7-23 \% \\
\mathrm{CA}(400 \mathrm{MPa} / 5-25 \mathrm{~min} ; 500 \\
\mathrm{MPa} / 5-15 \mathrm{~min}) ; \text { from } \approx \text { to } \downarrow 23 \% \\
\text { T.F3OLs/PACs } \\
\text { ATs: } \approx \text { cy-3-glu, } \approx \text { pel-3-glu, } \\
\approx \text { pel-3-rut, } \approx \text { T.ATs (all HPP } \\
\text { conditions) } \\
\text { EA: } \downarrow 2-37 \% \text { (400MPa/20 min; 500 } \\
\text { MPa/5, } 20 \mathrm{~min} ; 600 \mathrm{MPa} / 10,20, \\
25 \mathrm{~min})\end{array}$ & - & $\begin{array}{l}\text { TT } \\
\uparrow F 3 O L s / P A C s: \text { extraction from the achenes } \\
\text { favored by TT } \\
\downarrow \text { ATs: condensation reactions with other } \\
\text { phenols } \rightarrow \text { browning; PPO and POD } \\
\text { oxidation } \\
\uparrow E A: \text { release from the achenes; ETs } \\
\text { hydrolysis } \\
\text { HPP } \\
\downarrow \text { F3OLs/PACs: no complete enzyme } \\
\text { inactivation (PPO and POD) } \rightarrow \text { oxidation }\end{array}$ & [44] \\
\hline
\end{tabular}


Table 1. Cont

\begin{tabular}{|c|c|c|c|c|c|c|}
\hline Material & Treatment: Conditions & Storage & $\begin{array}{l}\text { Impact of Processing Conditions } \\
\text { on Polyphenols }\end{array}$ & $\begin{array}{c}\text { Impact of Storage } \\
\text { Conditions on Polyphenols } b\end{array}$ & Mechanisms & Ref. \\
\hline $\begin{array}{l}\text { Clear and cloud } \\
\text { strawberry juices }\end{array}$ & $\begin{array}{l}\mathrm{TT}(\mathrm{SB}): \\
100^{\circ} \mathrm{C} / 1 \mathrm{~min} \\
\mathrm{HPP}: \\
600 \mathrm{MPa} / 4 \text { min at } 43^{\circ} \mathrm{C}\end{array}$ & $\begin{array}{l}6 \text { months } \\
\text { at } 4 \text { and } 25^{\circ} \mathrm{C}\end{array}$ & - & $\begin{array}{l}\text { Clear juice at } 4{ }^{\circ} \mathrm{C} \\
\text { ATs: } \downarrow 10 \% \text { cy-3-glu, } \downarrow 6 \% \\
\text { pel-3-glu, } \downarrow 9 \% \text { pel-3-rut; } \downarrow 7 \% \\
\text { T.ATs } \\
\text { Cloudy juice at } 4{ }^{\circ} \text { CATs: } \downarrow 26 \% \\
\text { cy-3-glu, } \downarrow 33 \% \text { pel-3-glu, } \downarrow 21 \% \\
\text { pel-3-rut; } \downarrow 30 \% \text { T.ATs } \\
\text { Clear and cloudy juice at } 25 \\
{ }^{\circ} \text { C: } \\
\text { T.ATs: } \downarrow>95 \%\end{array}$ & $\begin{array}{l}\downarrow \text { ATs: PPO and POD oxidation; } \\
\text { condensation with other phenols } \rightarrow \\
\text { colorless compounds; oxidative } \\
\text { degradation of ascorbic acid (especially at } \\
\text { higher storage temperature) }\end{array}$ & [45] \\
\hline
\end{tabular}

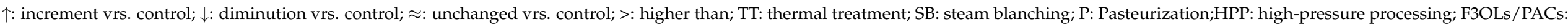

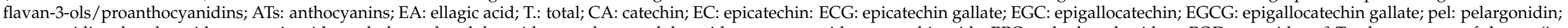

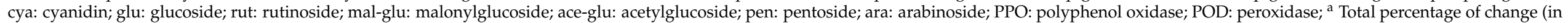

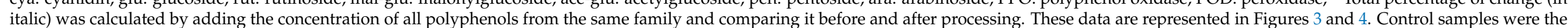

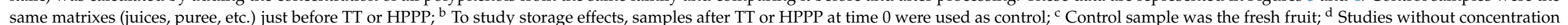
data to calculate the percentage of change (kinetics or graphs). 
Table 2. Effects of different TT and HPP treatments and storage on the main phenolic compounds in apple products.

\begin{tabular}{|c|c|c|c|c|c|c|}
\hline Material & Treatment: Conditions & Storage & $\begin{array}{c}\text { Impact of Processing Conditions on } \\
\text { Polyphenols }\end{array}$ & $\begin{array}{c}\text { Impact of Storage } \\
\text { Conditions on } \\
\text { Polyphenols }\end{array}$ & Mechanisms & Ref. \\
\hline $\begin{array}{l}\text { Apple } \\
\text { pureec }\end{array}$ & $\begin{array}{l}\text { TTwith } \mathrm{O}_{2}: \\
90{ }^{\circ} \mathrm{C} / 30 \text { min }+\mathrm{O} 2 \\
\text { TT } \varnothing \mathrm{O}_{2}: \\
90{ }^{\circ} \mathrm{C} / 30 \min \varnothing \mathrm{O}_{2}\end{array}$ & No & $\begin{array}{l}\text { TTwith } \mathrm{O}_{2} \\
\text { F3OLs/PACs: } \approx \text { EC; } \approx \text { procyanidin-dimer } 1 ; \\
\downarrow 45 \% \text { proanthocyanidin trimer; } \downarrow 62 \% \text { CA; } \\
\downarrow 30 \% \text { T.F3OLs/PACs } \\
\text { HCAs: } \downarrow 44 \% \text { chlorogenic acid } \\
\text { TT } \varnothing \mathrm{O}_{2} \\
\text { F3OLs/PACs: } \approx \text { EC, } \uparrow 35 \% \text { procyanidin-dimer } \\
1, \approx \text { proanthocyanidin trimer; } \approx C A ; \uparrow 7 \% \\
\text { T.F3OLs /PACs } \\
\text { HCAs: } \approx \text { chlorogenic acid }\end{array}$ & - & $\begin{array}{l}\downarrow \text { F3OLs/PACs and HCAs: } \\
\text { oxidation reactions during heating }\end{array}$ & {$[46]$} \\
\hline $\begin{array}{l}\text { Clear } \\
\text { apple } \\
\text { juice }\end{array}$ & $\begin{array}{l}\text { TT: } 25,35,45,55,65 \text { and } \\
75^{\circ} \mathrm{C} / 20 \mathrm{~min}\end{array}$ & No & $\begin{array}{l}\text { F3OLs/PACs: } \downarrow \text { EC and CA } \\
\text { HCAs: } \downarrow \text { chlorogenic acid }\end{array}$ & - & $\begin{array}{l}\downarrow \text { F3OLs/PACs and HCAs: } \\
\text { enzymatic oxidation }\end{array}$ & {$[47]^{\mathrm{d}}$} \\
\hline $\begin{array}{l}\text { Apple } \\
\text { juice }\end{array}$ & $\begin{array}{l}\text { TT1: } \\
71.7^{\circ} \mathrm{C} / 0.4 \mathrm{~min} \\
\text { TT2: } \\
90^{\circ} \mathrm{C} / 14.8 \mathrm{~min}\end{array}$ & No & $\begin{array}{l}\text { TT1 } \\
\text { F3OLs/PACs: } \uparrow 71 \% \text { T.F3OLs/PACs } \\
\text { HCAs: } \uparrow 244 \% \text { chlorogenic acid; } \uparrow 156 \% \\
\text { p-coumaoylquinic acid; } \uparrow 205 \% \text { T.HCAs } \\
\text { DHCs: } \uparrow 156 \% \text { phloretin xyloglucoside; } \uparrow 192 \% \\
\text { phloridzin; } \uparrow 165 \% \text { T.DHCs } \\
\text { FOLs: } \uparrow 39 \% \text { que-3-O-gal; } \uparrow 988 \% \text { que-3-O-hex; } \\
\uparrow 50 \% \text { que-3-O-xyl; } \uparrow 7 \% \text { que-3-O-rha; } \uparrow 33 \% \\
\text { que-3-O-pen; } \uparrow 49 \% \text { T.FOLs } \\
\text { TT2 } \\
\text { F3OLs/PACs: } \uparrow 1800 \% \text { T.F3OLs/PACs } \\
\text { HCAs: } \uparrow 1352 \% \text { chlorogenic acid; } \uparrow 389 \% \\
\text { p-coumaoylquinic acid; } \uparrow 925 \% \text { T.HCAs } \\
\text { DHCs: } \uparrow 752 \% \text { phloretin xyloglucoside; } \uparrow 808 \% \\
\text { phloridzin; } \uparrow 767 \% \text { total DHCs } \\
\text { FOLs: } \uparrow 67 \% \text { que-3-O-gal; } \uparrow 1113 \% \text { que-3-O-hex; } \\
\uparrow 92 \% \text { que-3-O-xyl; } \uparrow 14 \% \text { que-3-O-rha; } \uparrow 48 \% \\
\text { que-3-O-pen; } \uparrow 69 \% \text { T.FOLs }\end{array}$ & - & $\begin{array}{l}\uparrow F 3 \text { OLs / PACs: cleavage } \rightarrow \\
\text { release of dimers and monomers } \\
\uparrow F O L s: \text { release from cells walls; } \downarrow \\
\text { PPO activity } \\
\uparrow D H C s: \text { enhanced release from } \\
\text { peel and seeds } \\
\uparrow H C A s: \text { release from cells walls } \\
\text { favored by TT; enzyme } \\
\text { inactivation }\end{array}$ & {$[48]$} \\
\hline
\end{tabular}


Table 2. Cont

\begin{tabular}{|c|c|c|c|c|c|c|}
\hline Material & Treatment: Conditions & Storage & $\begin{array}{c}\text { Impact of Processing Conditions on } \\
\text { Polyphenols }\end{array}$ & $\begin{array}{l}\text { Impact of Storage } \\
\text { Conditions on } \\
\text { Polyphenols }\end{array}$ & Mechanisms & Ref. \\
\hline $\begin{array}{l}\text { Apple } \\
\text { juice } \\
\text { (2 years of } \\
\text { harvest) }\end{array}$ & TT: $85^{\circ} \mathrm{C}$ & $\begin{array}{l}360 \text { days } \\
4,20 \text {, and } \\
37^{\circ} \mathrm{C}\end{array}$ & - & $\begin{array}{l}4 \text { and } 20{ }^{\circ} \mathrm{C}: \\
\approx \sum \text { flavanols, DHCs, } \\
\text { FOLs, and phenol } \\
\text { carboxylic acids } \\
37^{\circ} \mathrm{C}: \\
\downarrow \sum \text { flavanols, DHCs, } \\
\text { FOLs, and phenol } \\
\text { carboxylic acids }\end{array}$ & $\begin{array}{l}\downarrow \sum \text { flavanols, DHCs, FOLs, and } \\
\text { phenol carboxylic acids: } \\
\text { Higly influenced by storage } \\
\text { temperature }\end{array}$ & {$[49]^{d}$} \\
\hline $\begin{array}{l}\text { Apple } \\
\text { juice }\end{array}$ & $\begin{array}{l}\mathrm{TT} 1: \\
98^{\circ} \mathrm{C} / 30 \mathrm{sec} \\
\mathrm{TT} 2: \\
98^{\circ} \mathrm{C} / 30 \mathrm{sec}\end{array}$ & No & $\begin{array}{l}\text { TT1 } \\
\text { F3OLs } / \text { PACs: } \downarrow 32 \% \text { CA; } \downarrow 31 \% \text { EC; } \downarrow 33 \% \text { EGC; } \\
\downarrow 43 \% \text { ECG; } \downarrow 18 \% \text { procyanidin B2; } \downarrow 18 \% \\
\text { T.F3OLs/PACs } \\
\text { FOLs: } \downarrow 25 \% \text { rutin; } \downarrow 50 \% \text { hyperin; } \downarrow 27 \% \text { que; } \\
\downarrow 32 \% \text { T.FOLs } \\
\text { DHCs: } \downarrow 18 \% \text { phloridzin } \\
\text { HCAs: } \downarrow 16 \% \text { chlorogenic acid } \\
\text { TT1+TT2 } \\
\text { F3OLs } / \text { PACs: } \downarrow 58 \% \text { CA; } \downarrow 56 \% \text { EC; } \downarrow 59 \% \text { EGC; } \\
\downarrow 70 \% \text { ECG; } \downarrow 37 \% \text { procyanidin B2; } \downarrow 48 \% \\
\text { T.F3OLs/PACs } \\
\text { FOLs: } \downarrow 64 \% \text { rutin; } \downarrow 86 \% \text { hyperin; } \downarrow 55 \% \text { que; } \\
\downarrow 63 \% \text { T.FOLs } \\
\text { DHCs: } \downarrow 48 \% \text { phloridzin } \\
\text { HCAs: } \downarrow 30 \% \text { chlorogenic acid }\end{array}$ & - & $\begin{array}{l}\downarrow \text { F3OLs/PACs: TT conditions } \\
\text { degraded heat-labile flavan-3-ols } \\
\downarrow \text { FOLs: due to the discard of peel } \\
\text { solids from the juice; the remaining } \\
\text { enzyme activity } \\
\downarrow \text { DHCs: due to thermal degradation }\end{array}$ & {$[50]$} \\
\hline $\begin{array}{l}\text { Apple sauce }{ }^{c} \\
\text { (12 varieties) }\end{array}$ & $\begin{array}{l}\text { TT1: } 95^{\circ} \mathrm{C} / 2 \mathrm{~min} \\
\text { TT2: } 95^{\circ} \mathrm{C} / 5 \mathrm{~min}\end{array}$ & No & $\begin{array}{l}\text { F3OLs/PACs: } \downarrow 20-85 \% \text { procyanidins oligomers; } \\
\downarrow 22-59 \% \text { CA; } \downarrow 13-74 \% \text { EC; } \downarrow 20-75 \% \\
\text { T.F3OLs/PACs } \\
\text { FOLs: from } \uparrow 6-63 \% \text { to } \downarrow 4-57 \% \text { T.FOLs; } \\
\text { DHCs: from } \downarrow 50 \% \text { to } \uparrow 54 \% \text { phloridzin; } \uparrow 1-1285 \% \\
\text { phloretin-2-xyloglucoside; from } \downarrow 8-14 \% \text { to } \\
\uparrow \% 8-325 \% \text { T.DHCs } \\
\text { HCAs: } \downarrow 1-47 \% 5^{\prime} \text {-caffeoylquinic acid (9 } \\
\text { varieties); } \uparrow 4-30 \% 5^{\prime} \text {-caffeoylquinic acid (4 } \\
\text { varieties); from } \downarrow 4-49 \% \text { to } \uparrow 7-27 \% \text { T. HCAs }\end{array}$ & & $\begin{array}{l}\uparrow \text { and } \downarrow \text { of phenols highly related to } \\
\text { the apple variety } \\
\downarrow \text { F3OLs/PACs: due to oxidation } \uparrow \\
\text { FOLs: diffusion of quercetin } \\
\text { glycosides from the peel to the } \\
\text { applesauce } \\
\uparrow \text { DHCs: diffusion from the seeds to } \\
\text { the applesauce } \\
\uparrow \text { HCAs: release from cells walls } \\
\text { favored by TT; Enzyme inactivation }\end{array}$ & [7] \\
\hline
\end{tabular}


Table 2. Cont

\begin{tabular}{|c|c|c|c|c|c|c|}
\hline Material & Treatment: Conditions & Storage & $\begin{array}{c}\text { Impact of Processing Conditions on } \\
\text { Polyphenols }{ }^{a}\end{array}$ & $\begin{array}{l}\text { Impact of Storage } \\
\text { Conditions on } \\
\text { Polyphenols }\end{array}$ & Mechanisms & Ref. \\
\hline $\begin{array}{l}\text { Cloudy apple } \\
\text { juice }^{\mathrm{c}}\end{array}$ & $\begin{array}{l}\text { TT: } \\
80-145^{\circ} \mathrm{C} / \text { over } 7200 \mathrm{sec}\end{array}$ & No & $\begin{array}{l}\text { F3OLs/PACs: } \downarrow 7 \text { procyanidin oligomers (mainly } \\
\text { B type); } \\
\uparrow \text { CA; } \uparrow E C ; \uparrow \text { dimeric compounds }\end{array}$ & - & $\begin{array}{l}\downarrow \text { F3OLs } / \text { PACs oligomers: } \\
\text { cleavage } \rightarrow \text { release of dimers and } \\
\text { monomers } \\
\downarrow \text { FOLs: glycosidic bond hydrolisis } \\
\text { in an acidic matrix }\end{array}$ & {$[51]^{\mathrm{d}}$} \\
\hline $\begin{array}{l}\text { Apple } \\
\text { juice }\end{array}$ & $\begin{array}{l}\text { HPP: } \\
300,300(x 3), \\
450,600 \mathrm{MPa} / 5 \mathrm{~min} \\
\text { at } 20{ }^{\circ} \mathrm{C}\end{array}$ & $\begin{array}{l}12 \text { weeks } \\
4{ }^{\circ} \mathrm{C}\end{array}$ & $\begin{array}{l}\text { F3OLs/PACs: } \approx \text { CA (all HPP); } \downarrow \text { EC (all HPP); } \\
\uparrow 8 \% \text { procyanidin B2 (300 and } 400 \mathrm{MPa}) ; \uparrow 18 \% \\
\text { procyanidin B2 (300 x3 and } 600 \mathrm{MPa}) ; \text { from } \approx \text { to } \\
\uparrow 8 \% \text { T. F3OLs/PACs } \\
\text { DHCs: } \approx \text { phloridzin }(300,300 \times 3,450 \mathrm{MPa} ; 600 \\
\mathrm{MPa}) \\
\text { FOLs: } \approx \text { que }(300,300 \times 3,450 \mathrm{MPa}) ; \uparrow 25 \% \text { que } \\
(600 \mathrm{MPa}) ; \uparrow 1-25 \% \text { T. FOLss } \\
\mathrm{HCAs:} \approx \text { chlorogenic acid }(300 \text { and } 300 \times 3 \mathrm{MPa}) ; \\
\uparrow 5 \% \text { chlorogenic acid }(450 \text { and } 600 \mathrm{MPa})\end{array}$ & $\begin{array}{l}\text { F3OLs } / \text { PACs: } \varnothing \text { C and } \\
\text { procyanidin B2 after } 6 \\
\text { weeks (all HPP); } \downarrow 77 \% \\
\text { EC (except in } 300 \mathrm{MPa}) \\
\text { FOLs: } \varnothing \text { quercetin after } 2 \\
\text { weeks (all HPP) } \\
\text { DHCs: } \downarrow 71-84 \% \\
\text { phloridzin (all HPP) } \\
\text { HCAs: } \downarrow 66-77 \% \\
\text { chlorogenic acid (300x3, } \\
450,600 \mathrm{MPa})\end{array}$ & $\begin{array}{l}\text { HPP: higher pressurization } \rightarrow \\
\text { higher extraction from apple } \\
\text { tissue } \\
\uparrow \text { FOLs: release from the disrupted } \\
\text { cell walls } \\
\text { Storage } \\
\downarrow \text { F3OLs/PACs and FOLs: } \\
\text { oxidation reactions }\end{array}$ & [52] \\
\hline
\end{tabular}


Table 2. Cont

\begin{tabular}{|c|c|c|c|c|c|c|}
\hline Material & Treatment: Conditions & Storage & $\begin{array}{c}\text { Impact of Processing Conditions on } \\
\text { Polyphenols }{ }^{\text {a }}\end{array}$ & $\begin{array}{l}\text { Impact of Storage } \\
\text { Conditions on } \\
\text { Polyphenols }\end{array}$ & Mechanisms & Ref. \\
\hline $\begin{array}{l}\text { Apples } \\
\text { (Spain } \\
\text { and Italy) }\end{array}$ & $\begin{array}{l}\mathrm{HPP}: \\
400,500,600 \mathrm{MPa} / 5 \mathrm{~min} \\
\text { at } 35^{\circ} \mathrm{C}\end{array}$ & No & $\begin{array}{l}\text { Spanish apples: } \\
400 \mathrm{MPa} \text { (best treatment) } \\
\text { F3OLs/PACs: } \approx \text { CA; } \approx \text { EC; } \approx \text { dimers; } \approx \text { trimers; } \\
\uparrow 4 \% \text { procyanidin B2; } \approx \text { T.F3OLs/PACs } \\
\text { FOLs: } \approx \text { que-3-rut; } \uparrow 35 \% \text { que-3-gal; } \uparrow 22 \% \\
\text { que-3-glu; } \uparrow 30 \% \text { que-3-ara; } \uparrow 32 \% \text { que-3-xyl; } \uparrow 33 \% \\
\text { que-3-rha; } \uparrow 30 \% \text { T.FOLs } \\
\text { DHCs: } \approx \text { phloridzin; } \downarrow 9 \% \\
\text { phloretin-2-xyloglucoside; } \downarrow 2 \% \text { T.DHCs } \\
\text { HCAs: } \downarrow 44 \% \text { chlorogenic acid; } \downarrow 9 \% \\
\text { neochlorogenic acid; } \downarrow 10 \% \text { cryptochlorogenic } \\
\text { acid; } \downarrow 17 \% \text { coumaroyl quinic acid; } \downarrow 39 \% \text { T. HCAs } \\
\text { 500-600 MPa } \\
\text { F3OLs/PACs: } \approx \text { CA; } \downarrow 8-13 \% \text { procyanidin B2; } \\
\approx \text { trimers; } \approx \text { dimers; } \downarrow 11-17 \% \text { EC; } \downarrow 11 \% \\
\text { T.F3OLs/PACs } \\
\text { FOLs: } \downarrow 40-50 \% \text { que-3-rut; } \downarrow 33-53 \% \text { que-3-gal; } \\
\downarrow 24-46 \% \text { que-3-glu; } \downarrow 3-16 \% \text { que-3-ara; } \downarrow 6-23 \% \\
\text { que-3-xyl; } \downarrow 15 \% \text { que-3-rha; } \downarrow 16-33 \% \text { T.FOLs } \\
\text { DHCs: } \downarrow 16-20 \% \text { phloridzin; } \downarrow 14-17 \% \\
\text { phloretin-2-xyloglucoside; } \downarrow 15-19 \% \text { T.DHCs } \\
\text { HCAs: } \downarrow 15-24 \% \text { chlorogenic acid; } \downarrow 4 \% \\
\text { neochlorogenic acid; } \downarrow 12-14 \% \text { cryptochlorogenic } \\
\text { acid; } \downarrow 12-19 \% \text { coumaroyl quinic acid; } \downarrow 14-22 \% \text { T. } \\
\text { HCAs } \\
\text { Italian apples: } \\
600 M P a \text { (best treatment) }\end{array}$ & - & $\begin{array}{l}\text { Differences highly influenced by apple origin } \\
\downarrow \text { Oligomers PACs } \rightarrow \text { epimerization changes } \\
\text { and depolymerization } \\
\uparrow \text { FOLs: enhanced extraction by higher } \\
\text { permeability or disruption of cell walls } \\
\downarrow \text { FOLs: residual enzyme activity (PPO and } \\
\text { POD) } \rightarrow \text { oxidation }\end{array}$ & [12] \\
\hline
\end{tabular}


Table 2. Cont

\begin{tabular}{|c|c|c|c|c|c|c|}
\hline Material & Treatment: Conditions & Storage & $\begin{array}{c}\text { Impact of Processing Conditions on } \\
\text { Polyphenols a }\end{array}$ & $\begin{array}{l}\text { Impact of Storage } \\
\text { Conditions on } \\
\text { Polyphenols }\end{array}$ & Mechanisms & Ref. \\
\hline $\begin{array}{l}\text { Apples } \\
\text { (Spain } \\
\text { and Italy) }\end{array}$ & $\begin{array}{l}\mathrm{HPP}: \\
400,500,600 \mathrm{MPa} / 5 \mathrm{~min} \\
\text { at } 35^{\circ} \mathrm{C}\end{array}$ & No & $\begin{array}{l}\text { F3OLs/PACs: } \uparrow 30 \% \text { CA; } \uparrow 39 \% \text { procyanidin B2; } \\
\uparrow 70 \% \text { trimers; } \uparrow 242 \% \text { dimers; } \uparrow 45 \% \text { EC; } \uparrow 58 \% \\
\text { T.F3OLs/PACs } \\
\text { FOLs: } \uparrow 88 \% \text { que-3-rut; } \uparrow 107 \% \text { que-3-gal; } \uparrow 78 \% \\
\text { que-3-glu; } \uparrow 59 \% \text { que-3-ara; } \uparrow 68 \% \text { que-3-xyl; } \uparrow 61 \% \\
\text { que-3-rha; } \uparrow 75 \% \text { T.FOLs } \\
\text { DHCs: } \uparrow 67 \% \text { phloridzin; } \uparrow 51 \% \\
\text { phloretin-2-xyloglucoside; } \uparrow 63 \% \text { T.DHCs } \\
\text { HCAs: } \uparrow 31 \% \text { chlorogenic acid; } \uparrow 4 \% \\
\text { neochlorogenic acid; } \uparrow 5 \% \text { cryptochlorogenic acid; } \\
\uparrow 51 \% \text { coumaroyl quinic acid; } \uparrow 29 \% \text { T. HCAs } \\
400-500 M P a \\
\text { F3OLs/PACs: } \uparrow 2-13 \% \text { CA; } \downarrow 10-19 \% \text { procyanidin } \\
\text { B2; } \approx \text { trimers; } \uparrow 131-161 \% \text { dimers; } \downarrow 4-14 \% \text { EC; } \\
\approx T . F 3 O L s / P A C s \\
\text { FOLs: } \uparrow 24 \% \text { que-3-rut (400 MPa); } \uparrow 10-44 \% \\
\text { que-3-gal; } \downarrow 35 \% \text { que-3-glu (400 MPa); } \downarrow 7-21 \% \\
\text { que-3-ara; } \downarrow 7-25 \% \text { que-3-xyl; } \downarrow 7-23 \% \text { que-3-rha; } \\
\uparrow 5-29 \% \text { T.FOLs } \\
\text { DHCs: } \downarrow 15 \% \text { phloridzin; } \downarrow 1-14 \% \\
\text { phloretin-2-xyloglucoside; } \downarrow 11-\uparrow 8 \% \text { T.DHCs } \\
\text { HCAs: } \downarrow 14-17 \% \text { chlorogenic acid; } \downarrow 8-13 \% \\
\text { neochlorogenic acid; } \downarrow 8-16 \% \text { cryptochlorogenic } \\
\text { acid; } \downarrow 3-7 \% \text { coumaroyl quinic acid; } \downarrow 13-16 \% \text { T. } \\
\text { HCAs }\end{array}$ & - & $\begin{array}{l}\text { Differences highly influenced by apple origin } \\
\downarrow \text { Oligomers PACs } \rightarrow \text { epimerization changes and } \\
\text { depolymerization } \\
\uparrow \text { FOLs: enhanced extraction by higher permeability or } \\
\text { disruption of cell walls } \\
\downarrow \text { FOLs: residual enzyme activity (PPO and POD) } \rightarrow \\
\text { oxidation }\end{array}$ & [12] \\
\hline
\end{tabular}


Table 2. Cont

\begin{tabular}{|c|c|c|c|c|c|c|}
\hline Material & Treatment: Conditions & Storage & $\begin{array}{c}\text { Impact of Processing Conditions on } \\
\text { Polyphenols }{ }^{\text {a }}\end{array}$ & $\begin{array}{l}\text { Impact of Storage } \\
\text { Conditions on } \\
\text { Polyphenols }\end{array}$ & Mechanisms & Ref. \\
\hline $\begin{array}{l}\text { Apples } \\
\text { (Spain) }\end{array}$ & $\begin{array}{l}\text { HPP: } \\
400 \mathrm{MPa}-/ 5 \mathrm{~min} \\
\text { at } 35^{\circ} \mathrm{C}\end{array}$ & No & $\begin{array}{l}\text { F3OLs/PACs: } \approx \text { procyanidin B1, EC trimers and } \\
\text { tetramers; } \downarrow 4 \% \text { EC; } \uparrow 10 \% \text { CA, } \uparrow 4 \% \text { procyanidin } \\
\text { B2; } \uparrow 65 \% \text { EC-dimer; } \uparrow 3 \% \text { T.F3OLs/PACs } \\
\text { FOLs: } \uparrow 9 \% \text { que-3-rut; } \uparrow 35 \% \text { que-3-gal; } \uparrow 22 \% \\
\text { que-3-glu; } \uparrow 30 \% \text { que-3-ara; } \uparrow 32 \% \text { que-3-xyl; } \uparrow 33 \% \\
\text { que-3-rha; } \uparrow 30 \% \text { T.FOLs } \\
\text { DHCs: } \approx \text { phloridzin; } \downarrow 9 \% \\
\text { phloretin-2-xyloglucoside; } \approx \text { T.DHCs } \\
\text { HCAs: } \downarrow 13 \% \text { chlorogenic acid; } \downarrow 9 \% \\
\text { neochlorogenic acid; } \downarrow 10 \% \text { cryptochlorogenic } \\
\text { acid; } \downarrow 17 \% \text { coumaroyl quinic acid; } \downarrow 12 \% \text { T. HCAs }\end{array}$ & & $\begin{array}{l}\downarrow \text { Oligomers PACs } \rightarrow \text { epimerization changes and } \\
\text { depolymerization }\end{array}$ & [53] \\
\hline $\begin{array}{l}\text { Cloudy } \\
\text { apple } \\
\text { juice }\end{array}$ & $\begin{array}{l}\text { HPP: } \\
600 \mathrm{MPa} / 5 \text { minat } 25^{\circ} \mathrm{C}\end{array}$ & $\begin{array}{l}12 \\
\text { weeks } \\
4{ }^{\circ} \mathrm{C}\end{array}$ & $\begin{array}{l}\text { F3OLs } / \text { PACs: } \approx \text { CA; } \downarrow 13 \% \text { EC; } \downarrow 45 \% \\
\text { procyanidin B1; } \downarrow 15 \% T . \text { F3OLs } / \text { PACs } \\
\text { DHCs: } \downarrow 18 \% \text { phloridzin } \\
\text { HCAs: } \approx \text { chlorogenic acid }\end{array}$ & $\begin{array}{l}\text { F3OLs/PACs: } \downarrow 92 \% \\
\text { procyanidin B1; } \\
\downarrow 61 \% ; \downarrow 15 \% \text { CA } \\
\text { DHCs: } \downarrow 71 \% \\
\text { phloridzin } \\
\text { HCAs: } \downarrow 53 \% \\
\text { chlorogenic acid }\end{array}$ & $\begin{array}{l}\downarrow \text { F3OLs/PACs: residual PPO and POD activity } \rightarrow \\
\text { oxidation } \\
\downarrow \text { DHCs: residual enzyme activity } \rightarrow \text { oxidation }\end{array}$ & [54] \\
\hline
\end{tabular}

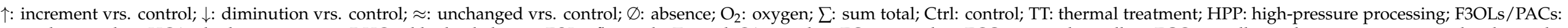

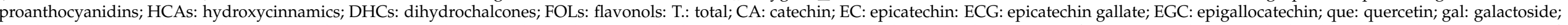

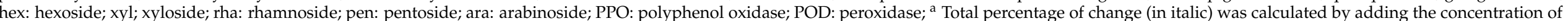

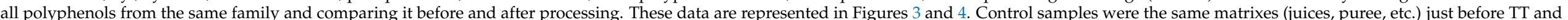

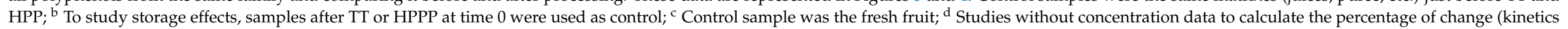
or graphs). 
Table 3. Impact of TT and HPP treatments on different apple and strawberry phenolic compounds. (F3OLs/PAC) flavan3-ols + proanthocyanidins; (ATs) anthocyanins; (EA) ellagic acid; (FOLs) flavonols; (DHCs) dihydrochalcones; (HCAs) hydroxycinnamic acids.

\begin{tabular}{|c|c|c|c|c|}
\hline & \multicolumn{2}{|c|}{ TT } & \multicolumn{2}{|c|}{ HPP } \\
\hline \multirow[b]{3}{*}{$\begin{array}{l}\text { F3OLs/ } \\
\text { PACs }\end{array}$} & \multicolumn{2}{|c|}{8 studies $/ 30$ trials } & \multicolumn{2}{|c|}{6 studies $/ 28$ trials } \\
\hline & \multirow[b]{2}{*}{$\begin{array}{ll}\text { - } & \text { Apple puree/juice } \\
\text { - } & \text { Pasteurized } \\
\text { - } & \text { Strawberries } \\
& \text { Strawberry } \\
& \text { pulp/puree }\end{array}$} & \multirow[b]{2}{*}{$\begin{array}{l}\text { - } \quad \text { Apple sauce/juice } \\
\text { - } \quad \text { Strawberry puree }\end{array}$} & Positive (13) & Negative (15) \\
\hline & & & $\begin{array}{ll}\text { - } & \text { Pressurized apples } \\
\text { - } & \text { Cloudy apple juice } \\
& \text { Strawberry puree }\end{array}$ & $\begin{array}{ll}\text { - } & \text { Strawberry } \\
& \text { puree/pulp } \\
\text { - } & \text { Pressurized apples } \\
\text { - } & \text { Apple juice }\end{array}$ \\
\hline \multirow[b]{3}{*}{ ATs } & \multicolumn{2}{|c|}{7 studies $/ 23$ trials } & \multicolumn{2}{|c|}{6 studies $/ 40$ trials } \\
\hline & \multirow[b]{2}{*}{ - $\quad$ Strawberry puree } & Negative (18) & Positive (16) & Negative (24) \\
\hline & & $\begin{array}{ll}\text { - } & \text { Pasteurized } \\
\text { strawberries } \\
\text { - } & \text { Strawberry } \\
\text { pulp/puree }\end{array}$ & $\begin{array}{l}\text { Strawberry } \\
\text { pulp/puree }\end{array}$ & $\begin{array}{l}\text { - Strawberry } \\
\text { pulp/puree }\end{array}$ \\
\hline \multirow[b]{3}{*}{ EA } & \multicolumn{2}{|c|}{5 studies/10 trials } & \multicolumn{2}{|c|}{3 studies $/ 27$ trials } \\
\hline & \multirow[b]{2}{*}{$\begin{array}{ll}\text { - } & \text { Strawberry } \\
\text { puree/pulp } \\
\text { - } & \text { Pasteurized } \\
\text { strawberries }\end{array}$} & Negative (4) & \multicolumn{2}{|c|}{$\begin{array}{l}3 \text { studies/27 trials } \\
\text { Positive (13) Negative (14) }\end{array}$} \\
\hline & & - $\quad$ Strawberry puree & - $\quad$ Strawberry puree & - $\quad$ Strawberry pulp \\
\hline \multirow[b]{3}{*}{ FOLs } & \multicolumn{2}{|c|}{3 studies $/ 17$ trials } & \multicolumn{2}{|c|}{3 studies $/ 11$ trials } \\
\hline & Positive (14) & Negative (3) & Positive (9) & Negative (2) \\
\hline & $\begin{array}{l}\text { - } \\
\text { - }\end{array}$ & $\begin{array}{l}\text { - } \\
\text { - }\end{array}$ & $\begin{array}{ll}\text { - } & \text { Pressurized apples } \\
\text { - } & \text { Apple juice }\end{array}$ & - $\quad$ Pressurized apples \\
\hline \multirow[b]{3}{*}{ DHCs } & \multicolumn{2}{|c|}{3 studies $/ 16$ trials } & \multicolumn{2}{|c|}{6 studies $/ 12$ trials } \\
\hline & Positive (12) & Negative (4) & Positive (6) & Negative (6) \\
\hline & $\begin{array}{ll}\text { - } & \text { Applesauce } \\
\text { - } & \text { Apple juice }\end{array}$ & $\begin{array}{l}\text { - } \\
\text { - }\end{array}$ & $\begin{array}{l}\text { - } \quad \text { Pressurized apples } \\
\text { - } \quad \text { Apple puree }\end{array}$ & $\begin{array}{ll}\text { - } & \text { Pressurized apples } \\
\text { - } & \text { Apple juice }\end{array}$ \\
\hline \multirow[b]{3}{*}{ HCAs } & \multicolumn{2}{|c|}{4 studies $/ 19$ trials } & \multicolumn{2}{|c|}{4 studies/12 trials } \\
\hline & Positive (5) & Negative (14) & Positive (5) & Negative (7) \\
\hline & - $\quad$ Apple puree/juice & $\begin{array}{ll}\text { - } & \text { Applesauce/puree } \\
\text { - } & \text { Apple juice }\end{array}$ & - $\quad$ Apple juice & $\begin{array}{ll}\text { - } & \text { Pressurized apples } \\
\text { - } & \text { Apple juice }\end{array}$ \\
\hline
\end{tabular}

F3OLs/PACs: proanthocyanidins; ATs: anthocyanins; EA: ellagic acid; FOLs: flavonols; DHCs: dihydrochalcones; HCAs: hydroxycinnamics.

For TT, a total of 8 studies with 30 trials including heat treatments from 70 to $98^{\circ} \mathrm{C}$ during 0.4 to 15 min provided concentration data to calculate percentages of change of total F3OLs/PACs. Increments (10 trials) from $4 \%$ to $1800 \%$ were observed on total F3OLs/PACs in apple puree and juice, pasteurized strawberry and strawberry pulp, and puree $[32,33,40,44,46,48]$. Conversely, reductions (20 trials) from $16 \%$ to $75 \%$ were reported 
in strawberry puree, applesauce, and apple juice $[7,33,46,50]$. Whereas for HPP, a total of six studies with 28 trials under the following pressurization conditions, 300 to $600 \mathrm{MPa}$ for 1 to $15 \mathrm{~min}$ at $22-35{ }^{\circ} \mathrm{C}$ were examined. Positive effects (13 trials) from no differences to $58 \%$ increments were observed in pressurized apples, apple juice, and strawberry puree $[12,40,44,52,53]$. However, decreases (15 trials) from $7 \%$ to $23 \%$ were reported in strawberry pulp, pressurized apples, and cloudy apple juice $[12,44,54]$ (Table 3 ).

Overall, HPP maintained the content of F3OLs/PACs closer to the fresh control in both fruits. In contrast, the impact of TT on the degree of change of F3OLs/PACs was positive or negative depending on the study, influenced by the matrix, the TT conditions, and the response of the fruit cultivar to the processing technology [6,7] (Figures 3A and 4). In addition, it is important to keep in mind that the type of extraction highly influences the reported F3OLs/PACs concentrations and the analytical method used.
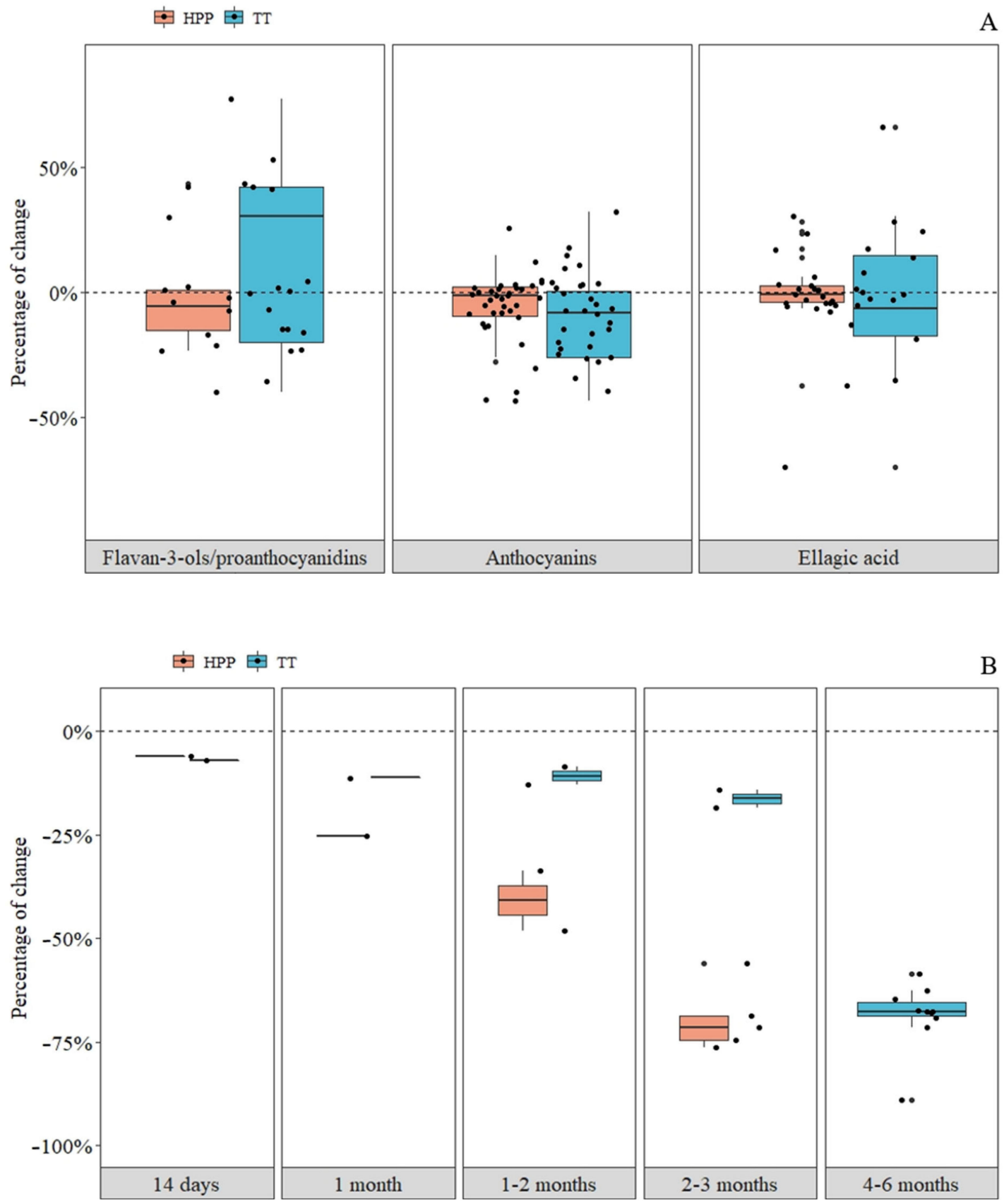

Figure 3. Impact of thermal treatment (TT) and high-pressure processing (HPP) on total flavan-3-ols/proanthocyanidins, 
anthocyanins, ellagic acid after different processing conditions (A), and on anthocyanins storage at $4-6{ }^{\circ} \mathrm{C}(\mathbf{B})$ in strawberry products. Each point represents the results of a trial. The percentages of change with the processing of each polyphenol family were calculated by adding the concentration of all polyphenols from the same family and comparing it before and after processing.

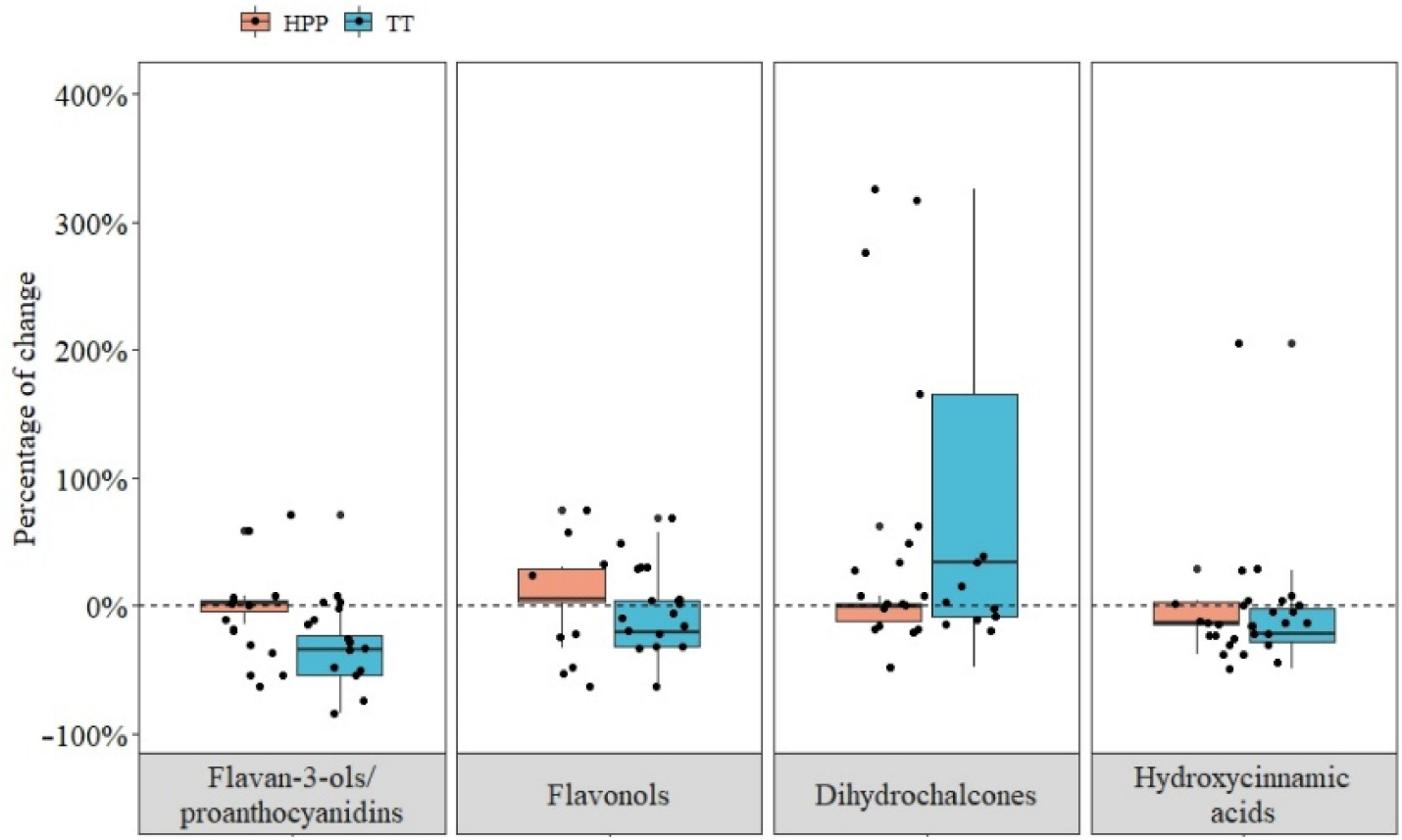

Figure 4. Impact of thermal treatment (TT) and high-pressure processing (HPP) on total flavan-3-ols/proanthocyanidins, flavonols, dihydrochalcones, and hydroxycinnamic acids after different processing conditions in apple products. Each point represents the results of a trial. The percentages of change with the processing of each polyphenol family were calculated by adding the concentration of all polyphenols from the same family and comparing it before and after processing. In TT, one trial with a percentage of change of F3OLs/PACs of $1800 \%$, another with a percentage of change in DHCs of $768 \%$, and another one with a percentage of change of HCAs of $925 \%$ were removed from the figures for scale reasons.

For TT, Stübler et al. [40] found a $122 \%$ increase in catechin and $33 \%$ in proanthocyanidin B1 levels after processing strawberry puree at $72{ }^{\circ} \mathrm{C}$ for $1 \mathrm{~min}$ [40]. An increase in catechin $(42 \%)$ was also observed in strawberry pulp treated at $70{ }^{\circ} \mathrm{C}$ for $2 \mathrm{~min}$ [44]. In the same direction, Oliveira et al. [31,32] reported increments on (+)-catechin, (-)-epigallocatechin, $(-)$-epicatechin, and (-)-epigallocatechin gallate levels in entire strawberries pasteurized at $90{ }^{\circ} \mathrm{C}$ for $5 \mathrm{~min}$. However, after 360 days of storage at $-20^{\circ} \mathrm{C}$ and 90 days at $23{ }^{\circ} \mathrm{C}$, the concentration of the monomers decreased compared to the content evaluated immediately after processing, although they were still higher than the fresh control [31,32]. Conversely, a general increase in polymeric PACs was observed in thermally treated $\left(90^{\circ} \mathrm{C} / 2 \mathrm{~min}\right)$ cloudy strawberry juice kept at 4 and $20^{\circ} \mathrm{C}$ for six months [37]. In this study, the PACs increment during storage could be explained by the protective effect exerted by the food matrix since cloudy juice contains pectin, which formed colloidal suspensions that limit their degradation. Consistent with the positive impact of TT on strawberry products, Alongi et al. [48] recorded a $71 \%$ increase in total flavan-3-ols after mild pasteurization $\left(71.7^{\circ} \mathrm{C} / 0.4 \mathrm{~min}\right)$ of apple juice and an $1800 \%$ increase after intense pasteurization $\left(90^{\circ} \mathrm{C} / 14.8 \mathrm{~min}\right)$. This vast increase was not represented in Figure 4 for reasons of scale. An increase in flavan-3-ol monomers together with a decrease in seven PAC oligomers was also reported in cloudy apple juice treated at $80-145^{\circ} \mathrm{C}$ for $120 \mathrm{~min}$ [51]. The positive effects observed in flavan-3ols were attributed to the heat treatment, which could have promoted the increase in these flavan-3-ol monomers, aiding in the release from the cellular tissue or favoring the cleavage of complex PACs molecules into their structural monomers [32,40,48,51]. On the contrary, 
other studies have reported reductions in F3OLs/PACs, mainly monomers, in strawberry puree after treatment at $85^{\circ} \mathrm{C} / 3 \mathrm{~min}$ [33], in clear apple juice treated $20 \mathrm{~min}$ at different temperatures $\left(25-75{ }^{\circ} \mathrm{C}\right)$ [47] and in apple juice after pre-pasteurization and pasteurization process $\left(98^{\circ} \mathrm{C} / 3 \mathrm{sec}\right)$ [50]. A wide degradation range (20-75\%) of total F3OLs/PACs was also observed in pasteurized applesauce from 12 varieties, showing the influence of the variety in the thermal treatment effects [7]. PACs degradation might be explained either by oxidation reactions due to insufficient inactivation of enzymes or because the monomers released into the food matrix are more prone to non-enzymatic reactions after processing and storage [32]. Kim et al. [46] proposed that not only heating $\left(90^{\circ} \mathrm{C}\right.$ for $\left.30 \mathrm{~min}\right)$ but also the presence of oxygen during processing affected the decreases observed for flavan-3-ols in apple puree.

As mentioned before, in general, HPP showed minor variation in F3OLs/PACs levels compared to control. In the case of strawberry puree pressurized at $600 \mathrm{MPa}$ for $1 \mathrm{~min}$, Stübler et al. [40] detected a rise of $19 \%$ on procyanidin B1 and $68 \%$ on catechin, which could be the result of the release of phenols to the food matrix from the disrupted cell walls. Similarly, Szczepańska et al. [52] registered about $8 \%$ increments in procyanidin B2 after 300 and $450 \mathrm{MPa}$, and $18 \%$ in apple juices subjected to $600 \mathrm{MPa}$ and multipulsed pressurization ( $300 \mathrm{MPa} \times 3$ pulses). An explanation for these results could be that higher pressurization induced a better extraction of procyanidin B2 from the tissue. However, catechin and epicatechin concentration decreased after all HPP treatments, and after 12 weeks of storage at $4{ }^{\circ} \mathrm{C}$, procyanidin $\mathrm{B} 2$ and catechin were not detected in all the pressurized juices [52]. Similarly, Cao et al. [44] reported lower catechin concentrations (4-23\%) after HPP (at 400, 500, and $600 \mathrm{MPa}$ for 5, 10, 15, 20, and $25 \mathrm{~min}$ ) than in the fresh strawberry pulp. Another study also found significant decreases of $13 \%$ and $45 \%$ on epicatechin and procyanidin B in cloudy apple juice after HPP (600 MPa for 5 min at $\left.25^{\circ} \mathrm{C}\right)$. However, no significant differences were reported on catechin [54]. Further degradations were reported for catechin, epicatechin, and procyanidin B1 after 12 weeks of storage at $4{ }^{\circ} \mathrm{C}$. The highest degradation was recorded for procyanidin B1 (92\%), which was not detected after week 4 . In general, the degradation of flavan-3-ol monomers, oligomers, and polymers after pressurization and subsequent storage might be attributed to insufficient inactivation of PPO and POD, which led to oxidation reactions and brown polymer formation. As occurred with the TT, apple origin and variety showed a high impact on the processing effect by HPP. Fernández-Jalao et al. [12] reported a different behavior after pressurization in Spanish and Italian apples. In Spanish apples, $400 \mathrm{MPa}$ showed increases of $4 \%$ in procyanidin B2, whereas 500 and $600 \mathrm{MPa}$ resulted in degradation of all F3OLs/PACs. In apples from Italy, pressurization at $600 \mathrm{MPa}$, resulted in increments of $30 \%$ in catechin, 39\% in procyanidin $\mathrm{B} 2,45 \%$ in epicatechin, $70 \%$ in trimers, and $240 \%$ in epicatechin dimers.

\subsection{Effects on the Stability of Anthocyanins in Strawberry Products}

Anthocyanins (ATs) are water-soluble compounds found in different tissues of the plant, such as leaves, roots, flowers, and fruits [55]. These play an essential role in the sensory attributes of food products, as they are responsible for the characteristic red, purple, and blue coloration in fruits [56]. Anthocyanins can be found as aglycones or glycosylated derivatives. The six most frequently found in foods are pelargonidin, cyanidin, malvidin, delphinidin, petunidin, and peonidin [57]. The highest AT levels are found in berries, currants, grapes, and tropical fruits [58]. In the case of fresh strawberries, AT concentrations from 3.7 to $64.9 \mathrm{mg} / 100 \mathrm{~g}$ FW have been reported $[3,6]$. Numerous in vitro and in vivo studies have recognized the potential effect on preventing neurodegenerative and cardiovascular diseases and antioxidant, anti-inflammatory, anti-obesity, anti-diabetic, and chemopreventive properties [59-61].

For TT, 7 studies with 23 trials comprising temperatures from 70 to $100{ }^{\circ} \mathrm{C}$ for 1 to $15 \mathrm{~min}$ provided concentration data to calculate percentages of change of total ATs. Reductions (18 trials) from 5\% to $44 \%$ were observed in pasteurized strawberry, strawberry 
purees and pulp $[32,33,36,41,42,44]$. On the contrary, other authors reported increments (5 trials) from $2 \%$ to $32 \%$ in strawberry puree $[33,40]$.

Regarding HPP, a total of 6 studies, including 40 trials with pressurization conditions from 100 to $600 \mathrm{MPa}$ for 1 to $20 \mathrm{~min}$ at $0-50{ }^{\circ} \mathrm{C}$, were analyzed. Degradations (24 trials) from $7 \%$ to $28 \%$ were reported in strawberry pulp and puree $[38,39,41,44]$. In contrast, positive effects (16 trials), from no differences to $15 \%$ increase, were recorded in the same products $[38,40,44]$ (Table 3$)$.

In general, just after pressurization, the levels of ATs were preserved close to those found in fresh strawberries. In contrast, a more significant impact was observed in the degree of change of ATs due to TT, with a downward trend in both cases (Figure 3A).

Since ATs are very chemically sensitive compounds, most of the studies registered the degradation of ATs after processing and storage. For instance, pasteurization of entire strawberries at $90{ }^{\circ} \mathrm{C}$ for $5 \mathrm{~min}$ resulted in a reduction of 5-35\% of cyanidin-3-glucoside, pelargonidin-3-rutinoside and pelargonidin-3-glucoside [31,32]. Higher degradations of $87-92 \%$ of these ATs were observed during 90 days of storage at $23{ }^{\circ} \mathrm{C}$ [32]. Similar results with losses of around $90-93 \%$ were observed after storage for eight weeks at $25^{\circ} \mathrm{C}$ of strawberry puree treated at $100{ }^{\circ} \mathrm{C}$ for $10 \mathrm{~min}$ [35]. In another study, the storage of strawberry puree (heated at $90{ }^{\circ} \mathrm{C}$ for $15 \mathrm{~min}$ ) during 12 weeks at $6{ }^{\circ} \mathrm{C}$ led to a mean degradation of about $19 \%$ of these ATs [43]. Degradation of total ATs (22\%) was also observed in the strawberry pulp after heating at $70{ }^{\circ} \mathrm{C}$ for $2 \mathrm{~min}$ [44] and in strawberry puree pasteurized $90{ }^{\circ} \mathrm{C}$ for $15 \mathrm{~min}$ (44\% of reduction) [41]. In general, degradation of ATs can follow three possible processes; cleavage of covalent bonds, polymerization and derivatization $[32,36,41,43]$. Moreover, this decrease could be partially caused by condensation reactions of ATs with other phenolics to produce colored polymeric pigments, resulting in strawberry pulp browning.

Interestingly, many authors have reported different degradation percentages depending on the strawberry variety, concluding that the effect of TT and subsequent storage on ATs also depends on the response of the fruit variety to the treatment [36,37,42]. The stability of ATs is also highly influenced by the food matrix properties. High $\mathrm{pH}$ and ascorbic acid content in the food matrix accelerates the degradation rate of ATs [43,62-64].

Although overall TT led to degradation on ATs, some studies reported a positive impact after processing. Garzoli et al. [33] reported a slightly higher content (2-18\%) on total ATs in pasteurized puree $\left(85^{\circ} \mathrm{C} / 3 \mathrm{~min}\right)$ compared with fresh puree. In agreement, Stübler et al. [40] showed that heat-treated $\left(72{ }^{\circ} \mathrm{C}\right.$ for $\left.1 \mathrm{~min}\right)$ strawberry puree incremented the individual ATs as follows: cyanidin-3-O-glucoside (40\%), pelargonidin-3-O-glucoside $(26 \%)$, pelargonidin-3-O-rutoside (22\%), pelargonidin-3-O-malonyl-glucoside $(34 \%)$, and pelargonidin-3-O-acetylglucoside (39\%). These increments in ATs might be attributed to a higher extraction of ATs from the matrix due to the heat treatment [40]. As mentioned before, and can be observed in Figure 3A, the changes in ATs content after HPP were minor compared with TT. Some studies reported no significant changes after pressurization. For instance, Bodelón et al. [38] found no significant differences in ATs levels in strawberry puree after HPP at 100, 200, 300, and $400 \mathrm{MPa}$ at $20^{\circ} \mathrm{C}$ compared with the untreated puree. However, a slightly higher decrease in ATs was observed in the puree pressurized at $50{ }^{\circ} \mathrm{C}$ compared with the untreated control. In line with this, the levels of individual and total ATs in strawberry pulp showed no significant changes after HHP treatments, regardless of the applied pressures or treatment times [44]. These results further support the idea that ATs were stable after pressurization. Stübler et al. [40] reported minor non-significant increases $(8-12 \%)$ on all the soluble individual ATs, in strawberry puree after pressurization at $600 \mathrm{MPa}$ for $1 \mathrm{~min}$ at room temperature. One explanation for this apparent increase in ATs could be the release of these compounds from the intact cells to the surrounding matrix. In other works, slight but significant decreases of about $7 \%$ on total ATs were reported in strawberry puree after $\mathrm{HPP}$ at 300 and $500 \mathrm{MPa}$ for 1,5 , and $15 \mathrm{~min}$ at $0{ }^{\circ} \mathrm{C}$. Under the same pressurization conditions combined with $50{ }^{\circ} \mathrm{C}$, there was a degradation of $14 \%$ on ATs [41]. In the samples pressurized without heat treatment, the oxidative enzyme activity 
(PPO and POD) did not change significantly, leading to oxidation reactions and thus ATs degradation. However, the higher degradation of ATs in the pressurized puree combined with heat treatment might be due to the formation of colorless chalcones and reduction in flavylium cations and quinoid bases as a consequence of the thermal treatment. In another study, significant losses of 15 and $21 \%$ of total ATs were recorded in strawberry puree from two-year crops treated with HPP at 300 and $600 \mathrm{MPa}$ for $15 \mathrm{~min}$ at $50{ }^{\circ} \mathrm{C}$ [39]. In the same line, Terefe et al. [42] also reported losses of ATs after HPP at $600 \mathrm{MPa}$ for $5 \mathrm{~min}$ at $20^{\circ} \mathrm{C}$ at different percentages depending on the variety: Camarosa (22-28\%), Ruby Gem (27-42\%), and Festival varieties (17-20\%). Similar behavior for all varieties was observed after three months of refrigerated storage with losses between $62 \%$ and $77 \%$. Losses up to $69 \%$ were also observed when pressurized strawberry puree $\left(500 \mathrm{MPa}\right.$ for $15 \mathrm{~min}$ at $\left.50^{\circ} \mathrm{C}\right)$ was stored for 12 weeks at $6{ }^{\circ} \mathrm{C}$ showing that even HPP in combination with temperature was not enough to inactivate enzymes and led to a significant degradation during storage [43].

The effect of refrigerated storage $\left(4-6{ }^{\circ} \mathrm{C}\right)$, from 14 to 180 days, on total ATs after different TT and HPP conditions is presented in Figure 3B. Storage at $4-6{ }^{\circ} \mathrm{C}$ after TT was examined in three studies $[37,42,43]$. Two studies were discussed to analyze the effect of storage after HPP, but only one used HPP without heat treatment [42], and the other combined HPP with heat treatment of $50{ }^{\circ} \mathrm{C}$ [43]. The losses of ATs during TT and HPP storage were progressive with the time, observing, in general, higher losses in the storage of HPP products. Storage from three to six months after HPP was only reported in a study with clear and cloudy strawberry juices that were not included in the figure because samples were also subjected to a previous blanching process at $100^{\circ} \mathrm{C} / 1 \mathrm{~min}$ in order to inactive enzymes [45].

\subsection{Effects on the Stability of Ellagic Acid in Strawberry Products}

Ellagitannins (ETs) constitute complex molecules with variable water solubility structured by one or more hexahydroxydiphenoyl (HHDP) moieties, which could be hydrolyzed to release ellagic acid (EA) [65]. ETs and EA are predominantly found in pomegranates, berry fruits, oak-aged red wine, tropical fruits, and nuts. ETs concentration in strawberries ranges from 7.18 to $28.85 \mathrm{mg} / 100 \mathrm{~g} \mathrm{FW}$ and EA between 0.9 and $14.8 \mathrm{mg} / 100 \mathrm{~g}$ FW [3,5] with a more significant contribution from achenes than from flesh $[5,66]$. The potential health benefits of ETs and EA are associated with the metabolites (urolithins) produced by the human gut microbiota $[67,68]$. The biological functions attributed to urolithins comprise anti-oxidant, neuroprotective, anti-microbial, anti-inflammatory, and anticancer properties $[67,69,70]$. Notably, there were no differences in urolithins' production and urinary excretion between volunteers ingesting either fresh strawberries or thermally treated puree [71].

For TT, 5 studies with 10 trials including temperatures from 55 to $90{ }^{\circ} \mathrm{C}$ for 1 to $15 \mathrm{~min}$ provided concentration data to calculate percentages of change of EA. Increases (6 trials) from $8 \%$ to $66 \%$ were observed in pasteurized strawberries, strawberry pulp, and strawberry purees [32,40,41,44]. In contrast, only Álvarez-Fernández et al. [34] reported a slight EA degradation (4 trials) from $8 \%$ to $35 \%$ in strawberries. Whereas for HPP, 3 studies with 27 trials with pressurization conditions ranging from 100 to $600 \mathrm{MPa}$ for 1 to $25 \mathrm{~min}$ at $0-50{ }^{\circ} \mathrm{C}$ were analyzed. Increments ( 13 trials) from non-differences to $28 \%$ were reported in strawberry puree [40,41], whereas 2 to $37 \%$ decreases (14 trials) were observed in strawberry pulp [44], showing that the increases observed were most probably due to the enhanced extraction from the achenes (Table 3).

In general, TT and HPP influenced the level of EA both positively and negatively depending on the conditions. Still, the most significant increases were observed as a result of heat treatment (Figure 3A).

For TT, Cao et al. [44] reported a 17\% increment in EA content after heating strawberry pulp at $70{ }^{\circ} \mathrm{C}$ for $2 \mathrm{~min}$. The rise of EA levels after processing could have resulted from the hydrolysis of ETs and the release from the cellular structures. In agreement, pasteurization of strawberry puree at $90{ }^{\circ} \mathrm{C}$ for $15 \mathrm{~min}$ led to an increase of $31 \%$ on EA [41], and only a 
limited increase of $8 \%$ was observed when a milder TT of $72{ }^{\circ} \mathrm{C}$ for 1 min was applied [40]. Likewise, pasteurized $\left(90^{\circ} \mathrm{C} / 5 \mathrm{~min}\right)$ strawberries had $143 \%$ higher levels of EA when compared with fresh fruit. However, after 360 days of storage at $-20{ }^{\circ} \mathrm{C}$, EA decreased $65 \%$ compared to the control [31]. Another study showed a zero-order kinetic model for EA degradation, with a final reduction of $32 \%$ in pasteurized strawberries stored for 90 days at $23{ }^{\circ} \mathrm{C}$. Degradation due to storage might be due to increased exposure of EA released from cell walls, leading to non-enzymatic oxidation reactions [32]. On the other hand, during storage of pasteurized strawberry pure $\left(90^{\circ} \mathrm{C} / 15 \mathrm{~min}\right)$ at $6{ }^{\circ} \mathrm{C}$ for 12 weeks, EA levels raised until week 10 and then slightly decreased toward the end of storage [43]. An explanation for the increase in EA during storage might be attributed to progressive release from high molecular weight ETs present in the puree [43]. Although the general trend is to increase EA after TT, Álvarez-Fernández et al. [34] reported decreases in EA (8-35\%) during the strawberry puree processing and in the final product in the 2011 and 2012 harvest.

For HPP, Cao et al. [44] reported significant losses from $2 \%$ to $37 \%$ on EA levels in strawberry puree after HPP at $400 \mathrm{MPa}$ for 5, 10, 15, 20, and $25 \mathrm{~min}$, and $500 \mathrm{MPa}$ for 5,10 , and $15 \mathrm{~min}$ at room temperature. However, a minor and non-significant decrease of 3\% EA was observed in strawberry puree pressurized at $600 \mathrm{MPa}$ for $1 \mathrm{~min}$ at room temperature [40]. In agreement, Marszalek et al. [41] also reported that the levels of EA in fresh strawberry puree did not change significantly after HPP at 300 and $500 \mathrm{MPa}$ for 1, 5, and $15 \mathrm{~min}$ at $0{ }^{\circ} \mathrm{C}$. However, when combining the same pressurization conditions at $50{ }^{\circ} \mathrm{C}$, a significant increase of $28 \%$ EA concentration was observed [41]. These increments could be due to a release of EA from ETs due to the combination of HPP with temperature [72]. An EA increase of $43 \%$ was reported when strawberry puree pressurized at $500 \mathrm{MPa}$ for $15 \mathrm{~min}$ at $50{ }^{\circ} \mathrm{C}$ was stored for 12 weeks at $6^{\circ} \mathrm{C}$ [43].

\subsection{Effects on the Stability of Flavonols in Apple Products}

Flavonols (FOLs) are plant secondary metabolites that could be found in foods as aglycones or much more frequently as glycosidic conjugates [73]. The most commonly found in foodstuff are quercetin, kaempferol, myricetin, and isorhamnetin [74]. Although these are present in various fruits and vegetables, the major sources of FOLs are capers, saffron, onion, and tea $[74,75]$. Among the FOLs, quercetin glycosides are the most frequently found in apples. These are located mainly in the peel in concentrations from 5.3 to $119.7 \mathrm{mg} / 100 \mathrm{~g}$ FW [4]. However, some studies also reported minor amounts in the flesh $[9,13]$. Numerous studies have reported the benefits of FOL consumption on preventing cardiovascular diseases, diabetes, inflammation, viral infections, neurodegeneration, and cancer [76-78].

For TT, 3 studies with 17 trials including temperatures from 71 to $98^{\circ} \mathrm{C}$ during 0.4 to $15 \mathrm{~min}$ provided concentration data to calculate percentages of change of total FOLs. Increments ranging from $4 \%$ to $69 \%$ were observed in five trials with apple juice and applesauce $[7,48]$. In contrast, reductions (12 trials) from $32 \%$ to $63 \%$ were detected in other studies with apple juice and applesauce [7,50]. A total of 3 studies with 11 trials under the following pressurization conditions, 300 to $600 \mathrm{MPa}$ for 5 to $15 \mathrm{~min}$ at $22-35^{\circ} \mathrm{C}$, were analyzed after HPP. Positive effects ranged between $1 \%$ and $75 \%$ in nine trials after pressurization in entire apples and apple juice $[12,52,53]$. Conversely, decreases (2 trials) from $16 \%$ to $33 \%$ were also observed in pressurized apples [12] (Table 3).

Overall, HPP had positive effects on FOLs concentration. With TT, the results depended on the conditions and the apple varieties with a general trend to decrease their content (Figure 4).

For instance, FOLs contents increased $49 \%$ and $69 \%$ after mild $\left(71{ }^{\circ} \mathrm{C} / 0.4 \mathrm{~min}\right)$ and intense $\left(90^{\circ} \mathrm{C} / 14.8 \mathrm{~min}\right)$ pasteurization of apple juice, respectively, which indicates that thermal treatment favored the release of phenolic compounds and reduced PPO activity [48]. Additionally, the thermal treatment also increased the bioaccessibility of FOLs compared to the fresh sample [79]. On the contrary, pre-pasteurization $\left(98^{\circ} \mathrm{C} / 30 \mathrm{~s}\right)$ and pasteurization 
$\left(98^{\circ} \mathrm{C} / 50 \mathrm{sec}\right.$ ) of apple juice from Red Fuji variety reduced rutin (64\%), hyperin (86\%), and quercetin levels $(55 \%)$. A possible explanation for this decline might be the incomplete inactivation of PPO and POD enzymes by milder heat treatments [50]. The occurrence of these enzymes should be determined after the TT conditions. In another study with applesauce (12 varieties), it was impossible to calculate exactly the change percentage of FOLs after TT. Concentration before processing was reported separately in fresh flesh and peel [7]. In this case, $95 \%$ of flesh and $5 \%$ of peel were considered to calculate the concentration in the fresh fruit (control sample). Like in apple peel, six quercetin glycosides were found in applesauce in this order of importance: quercetin-3-galactoside $>$ quercetin-3-arabinopyranoside $>$ quercetin-3-rhamnoside $>$ quercetin-3-glucoside $>$ quercetin-3-xyloside $>$ quercetin-3rutinoside. After crushing, cooking $\left(95^{\circ} \mathrm{C} / 2 \mathrm{~min}\right)$, and pasteurization $\left(90^{\circ} \mathrm{C} / 5 \mathrm{~min}\right)$, different behavior was observed depending on the variety: increments of FOLs from $4 \%$ to $57 \%$ were observed in three varieties, and decreases from $6 \%$ to $63 \%$ were observed in 9 varieties. FOLs in the applesauce could result from the diffusion of quercetin derivatives from the peel during crushing or from the presence of small particles of peel retained in the sauce. Differences in the percentages between varieties could be explained by differences in the skin of apples, different structures, mechanical resistance, and cuticle thickness.

About HPP, no significant differences were reported in quercetin levels after apple juice pressurization at 300 and $450 \mathrm{MPa}$ for $5 \mathrm{~min}$ and in multi-pulsed HPP at $300 \mathrm{MPa} \times 3$ pulses, each $5 \mathrm{~min}$. However, after HPP treatment at $600 \mathrm{MPa}$ for $5 \mathrm{~min}$, a minor but significant increase from 0.73 to $0.91 \mathrm{mg} / \mathrm{L}(25 \%)$ was quantified for quercetin [52]. These results could be attributed to an enhanced extraction from the juice tissue due to the high pressurization. Quercetin was not detected after two weeks of storage at $4{ }^{\circ} \mathrm{C}$ in all the treatments, and this degradation might be attributed to residual enzyme activity, which led to oxidation reactions [52]. Increases (9-35\%) in different glycoside conjugates of quercetin were also found between the untreated and pressurized apples at $400 \mathrm{MPa}$ for $5 \mathrm{~min}$ [12] As for other phenolic compounds, FOLs stability also depends on the fruit variety, degree of ripening, and food matrix characteristics $(\mathrm{pH}$, sugar content, and presence/absence of oxygen), which influence the behavior of the enzymes [12,53]. In this sense, FernándezJalao et al. [12] concluded that individual and total FOLs levels after pressurization were affected by HPP conditions and the apple origin. In terms of total FOLs concentration, the best results were obtained after pressurization at $400 \mathrm{MPa}$ in Spanish apples, increasing $30 \%$. However in 500 and $600 \mathrm{MPa}$ led to significant reductions of quercetin-3-rutinoside (40-50\%), quercetin-3-galactoside (33-53\%) and quercetin-3-glucoside (24-46\%) [12]. The degradation of quercetin glycosides might result from oxidation reactions caused by the residual activity of PPO and POD. In Italian apples, all the pressurization treatments increased quercetin glycosides, but the highest increase of about $75 \%$ was reported for $\mathrm{HPP}$ at $600 \mathrm{MPa}$ [12]. The increase in individual and total FOLs content after HPP might be attributed to a change in the cell walls permeability and/or by disruption of the cell membranes, promoting a better extractability from cellular tissues [53].

\subsection{Effects on the Stability of Dihydrochalcones in Apple Products}

The dihydrochalcones (DHCs), phloretin and phloridzin, are characteristic compounds of apple and apple products. Since these compounds are exclusive of apples, these have been used to detect adulterations [80]. DHCs levels in entire apples ranged from 0.011 to $0.043 \mathrm{mg} / 100 \mathrm{~g}$ FW [11,12]. Although the DHCs could be found in flesh and peel, the highest concentration is located in the seeds, ranging from 24.1 to $86.4 \mathrm{mg} / 100 \mathrm{~g}$ FW [8]. Numerous studies have shown that both phloridzin and phloretin exert antibacterial, anti-inflammatory, antihyperglycemic, anti-diabetic and anticancer activities, and cardioprotective, neuroprotective hepatoprotective, and immunomodulatory properties [81-83].

For TT, 3 studies with 16 trials comprising thermal treatments from 71 to $98^{\circ} \mathrm{C}$ during 0.4 to $15 \mathrm{~min}$ provided concentration data to calculate percentages of change of total DHCs. Increments (12 trials) ranged from $8 \%$ to $767 \%$ in applesauce and apple juice [7,48]. Conversely, reductions (4 trials) from $8 \%$ to $48 \%$ in apple juice and applesauce were 
recorded [7,50]. For HPP, four studies with 12 trials at pressurization conditions from 300 to $600 \mathrm{MPa}$ for 5 to $15 \mathrm{~min}$ at $22-35{ }^{\circ} \mathrm{C}$ were analyzed. Positive effects ( 6 trials) showing from no differences to $63 \%$ increase were noted in pressurized apples and apple juice [12,52]. Conversely, reductions ( 6 trials) from $2 \%$ to $19 \%$ were reported for pressurized apples and cloudy apple juice $[12,53,54]$ (Table 3 ).

Overall, TT and HPP changed the content of DHCs of apple products (Figure 4) positively. These differences are mainly explained by the use of whole apples during crushing in industrial processing, which contributes to releasing these compounds from the peel and seeds [84].

For TT, Le Bourvellec et al. [7] reported high variability on change percentages of DHCs in applesauce depending on the variety. Increments up to $325 \%$ were observed in nine varieties after crushing, cooking $\left(95^{\circ} \mathrm{C} / 2 \mathrm{~min}\right)$ and pasteurization $\left(90^{\circ} \mathrm{C} / 5\right) \mathrm{min}$ compared with fresh control (calculated as $95 \%$ of flesh $+5 \%$ of peel). An explanation for the higher DHCs levels after processing could be the little peel and seed particles in the applesauce $[7,85]$. One interesting finding in this study was identifying a colorless phloridzin oxidation product, which indicated limited enzymatic oxidation due to processing. In line with this, Alongi et al. [48] detected increases of $165 \%$ and $767 \%$ on total DHCs in apple juice after mild $\left(71^{\circ} \mathrm{C} / 0.4 \mathrm{~min}\right)$ and intense $\left(90^{\circ} \mathrm{C} / 14.8 \mathrm{~min}\right)$ pasteurizations, respectively. These results confirm the association that crushing and heat treatment increase the release of phenols from peel and seeds. In contrast, Tian et al. [50] reported $18 \%$ and $48 \%$ reduction in phloridzin levels after pre-pasteurization $\left(98{ }^{\circ} \mathrm{C} / 30 \mathrm{sec}\right)$ and pasteurization $\left(98^{\circ} \mathrm{C} / 30 \mathrm{~s}\right)$ respectively, probably due to a thermal degradation.

For HPP, Szczepańska et al. [52] reported no significant differences in phloridzin concentration in apple juice pressurized at 300,450 , and $600 \mathrm{MPa}$, and multi-pulsed $300 \mathrm{MPa} \times 3$ pulses for $5 \mathrm{~min}$. However, after 12 weeks of refrigerated storage, phloridzin concentration was reduced between $71 \%$ and $84 \%$ depending on the HPP conditions. In another study, after HPP at $600 \mathrm{MPa}$ for $5 \mathrm{~min}$ at $25^{\circ} \mathrm{C}$, phloridzin levels changed from 48.8 to $40.2 \mathrm{mg} / \mathrm{L}$, representing losses of $18 \%$. After two weeks of storage at $4{ }^{\circ} \mathrm{C}, 51 \%$ decreases were observed, reaching up to $71 \%$ losses at the end of 12 weeks of storage [54]. As for FOLs, the degradation in DHCs was mainly due to HPP conditions that were insufficient to inactivate enzymes (PPO and POD). Therefore, the phenolic compounds underwent oxidation reactions. Fernández-Jalao et al. [12] indicated that the apple origin conditioned the effects of HPP on DHCs. In Spanish apples, 500 and $600 \mathrm{MPa}$ treatments led to reductions in phloridzin (16-20\%) and phloretin-2'-xylosylglucoside (14-17\%) levels. Conversely, in Italian apples, HPP at $600 \mathrm{MPa}$ increased $51 \%$ and $67 \%$ the phloretin- $2^{\prime}$ xylosylglucoside and phloridzin concentrations, respectively.

\subsection{Effects on the Stability of Hydroxycinnamic Acids in Apple Products}

Hydroxycinnamic acids (HCAs) are the major subgroup of phenolic acids and may occur either in their free or conjugated forms, including amides, esters, and glycosides [86]. The main aglycones identified in foodstuff are $p$-coumaric, caffeic, ferulic, and sinapic acids [87]. More frequently found in coffee, various fruits, some vegetables, and whole grains [88]. In apples, chlorogenic acid (5-O-caffeoylquinic) is the predominant compound. The HCA levels vary depending on the parts of the fruit. In apples, the flesh is the structure characterized for the higher levels of HCAs [89]. Ranges in whole apples were from 2.5 to $23.1 \mathrm{mg} / 100 \mathrm{~g} \mathrm{FW}[11,12]$. Whereas in apple flesh, HCAs levels varied from 0.7 to $14.3 \mathrm{mg} / 100 \mathrm{~g}$ FW $[9,10]$. Some potential health benefits have been documented from HCAs intakes, such as anti-microbial, anti-diabetic, antioxidant activity, prevention of cardiovascular and neurodegenerative diseases, and some cancer conditions $[87,90,91]$.

For TT, four studies with 19 trials comprising heat treatments from 71 to $98^{\circ} \mathrm{C}$ for 0.4 to $30 \mathrm{~min}$ provided concentrations data to calculate percentages of change of total HCAs. Increments (5 trials) ranged from $8 \%$ to $925 \%$ in apple sauce and apple juice [7,48]. On the other hand, reductions (14 trials) fluctuated from $4 \%$ to $49 \%$ in apple puree, sauce, and juice $[7,46,50]$. For HPP, four studies with 12 trials with pressurization conditions from 300 
to $600 \mathrm{MPa}$ for 5 to $15 \mathrm{~min}$ at 22 to $35^{\circ} \mathrm{C}$ were reviewed. Positive effects ( 5 trials) from no significant differences to increments up to $29 \%$ were recorded in entire apples and apple juice [12,52]. Conversely, losses (7 trials) varied from $12 \%$ to $39 \%$ in whole apples and cloudy apple juice $[10,53,54]$ (Table 3$)$.

In general, both TT and HPP caused degradation of HCAs after processing. Nevertheless, although a higher degree of degradation was observed after heat treatment, it also caused the most significant increase in two trials with apple juice (the highest one is not shown in the graph for scale reasons) (Figure 4).

For TT, Alongi et al. [48] identified the highest increases in HCAs after heat treatment, observed in both chlorogenic acid and $p$-coumaroylquinic acid. A total of $205 \%$ and $925 \%$ increments on total HCAs were reported after the mild $\left(71^{\circ} \mathrm{C} / 0.4 \mathrm{~min}\right)$ and intense $\left(90{ }^{\circ} \mathrm{C} / 15 \mathrm{~min}\right.$ ) pasteurization of apple juice, respectively. The increase in HCAs could result from the favoring effect of thermal treatment in enzyme inactivation and the release of chlorogenic acid from the cell walls to the food matrix $[7,48]$. These results corroborate the finding of De Paepe et al. [51], who concluded that phloretin-2'-O-glucoside and 3-Ocaffeoylquinic acid were thermal-resistant compounds in cloudy apple juice isothermally treated from 80 to $145{ }^{\circ} \mathrm{C}$ during $7200 \mathrm{~s}$. In contrast, Tian et al. [50] reported a 15\% and 30\% degradation of chlorogenic acid levels in apple juice after pre-pasteurization $\left(98{ }^{\circ} \mathrm{C} / 30 \mathrm{~s}\right)$ and pasteurization $\left(98^{\circ} \mathrm{C} / 50 \mathrm{sec}\right)$ respectively. In agreement with these results, a significant decrease of $44 \%$ in chlorogenic acid was recorded in apple puree heated at $90{ }^{\circ} \mathrm{C}$ for $30 \mathrm{~min}$ in the presence of oxygen after storage. However, no effect was observed in the heat-treated puree in the absence of oxygen [46], suggesting that oxidation reactions during heating are the main reason for the HCAs degradation [46,50]. In addition, Le Bourvellec et al. [7] reported that the higher differences on $5^{\prime}$-caffeoylquinic acid and $p$-coumaroylquinic acid after TT were due to apple variety. After cooking $\left(95^{\circ} \mathrm{C} / 2 \mathrm{~min}\right)$ and pasteurization $\left(90{ }^{\circ} \mathrm{C} / 5 \mathrm{~min}\right)$, nine varieties experienced reductions from $1 \%$ to $48 \%$ in $5^{\prime}$-caffeoylquinic acid, whereas increases of $8 \%, 4 \%$, and 30\% were observed in Golden Delicious, Freiberg, and Granny Smith varieties, respectively.

For HPP, Marszalek et al. [54] found no significant differences in chlorogenic acid concentration of cloudy apple juice due to pressurization at $600 \mathrm{MPa}$ for $5 \mathrm{~min}$ at $25^{\circ} \mathrm{C}$. However, there was a progressive degradation up to $53 \%$ after 12 weeks of storage at $4{ }^{\circ} \mathrm{C}$. Accordingly, Szczepańska et al. [52] reported no differences in chlorogenic acid levels after static $\mathrm{HPP}$ at $300 \mathrm{MPa}$ for $5 \mathrm{~min}$ and multi-pulsed pressurization $(300 \mathrm{MPa} \times 3$ pulses). However, a slight increase of around 5\% was observed on the pressurized juices at 450 and $600 \mathrm{MPa}$ for $5 \mathrm{~min}$. Refrigerated storage for 12 weeks produced substantial losses of chlorogenic acid (66-77\%). Reductions of 12\% for total HCAs were reported for apples pressurized at $400 \mathrm{MPa}$ for $5 \mathrm{~min}$ [53]. In another study, Fernández-Jalao et al. [12] reported that the HPP effect on individual and total HCAs was associated with the apple origin. In Spanish apples, pressurization reduced all individual HCAs, neochlorogenic, cryptochlorogenic, coumaroylquinic, and chlorogenic acids. The latter is the one that suffered the most significant degradations, with losses of $44 \%$ at $400 \mathrm{MPa}, 24 \%$ at $500 \mathrm{MPa}$, and $15 \%$ at $600 \mathrm{MPa}$. Whereas in Italian apples, HPP at $600 \mathrm{MPa}$ increased all the individual HCAs, resulting in a total increment of $29 \%$. In contrast, $\mathrm{HPP}$ at 400 and $500 \mathrm{MPa}$ reduced total HCAs by $16 \%$ and $13 \%$, respectively.

\section{Conclusions}

This review analyzed the effects of TT and HPP treatments on the phenolic compounds in strawberry (F3OLs/PACs, ATs, and EA) and apple (F3OLs/PACs, FOLs, DHCs, and HCAs) products. Our findings show that the effect on polyphenols content (positive or negative) was contingent upon the type of processing, type of fruit, polyphenol family, and the shelf-life conditions (time and temperature during storage) of the final product. The impact of TT relied mainly on the food matrix and the thermal stability of the different phenolic compounds. 
TT had positive effects in strawberry products, as was observed for F3OLs/PACs and EA. However, it had negative effects on the ATs content. These were due to the thermal instability of ATs and the enhanced extraction of EA and condensed tannins from achenes with thermal treatments. It is well known that ATs are heat-labile compounds, susceptible to oxidation and condensation reactions, which was confirmed in most of the studies where degradation of ATs was observed after TT. On the contrary, TT increased EA content due to the hydrolysis from ETs and release from the lignified matrix of the achenes.

In apple products, TT had positive effects, significantly promoting the release of FOLs and DHCs from peel and seeds. However, most of the studies observed a negative effect of F.OL/PACs, principally due to the extraction processes that quantified flavan-3-ols oligomers rather than monomers. Nevertheless, the final concentrations were variable depending on the variety, and the TT conditions applied. HPP treatments maintained the concentrations of phenolic compounds closer to those of the fresh or unprocessed samples regardless of the food matrix.

The impact of storage after TT and HPP had only been described for ATs in strawberry products. In general, TT has positive effects preserving better the ATs during storage. However, in the only study that examined the effect of HPP on ATs during storage, a negative effect was described showing a higher degradation than TT. Negative effects were also observed as the fast and pronounced degradation of FOLs, DHCs, and HCAs in apple products after HPP and storage.

The phenolic compounds' degradation after storage of HPP products could be due to the limitations in oxidative enzyme inactivation when thermal treatments were insufficient. From the industrial perspective, manufacturers aiming to preserve the natural content of polyphenols need to find the sweet spot between polyphenol stability and product shelf life. Further studies are recommended to compare how both technologies influence the content of the different families of polyphenols and their bioavailability and bioactivity.

Author Contributions: Conceptualization, L.M.S.-S. and F.A.T.-B.; methodology, L.M.S.-S. and R.G.V.; formal analysis, G.L.S.-O.; investigation, G.L.S.-O., R.G.-V., L.M.S.-S., and F.A.T.-B.; resources, L.M.S.-S. and F.A.T.-B.; data curation, G.L.S.-O. and R.G.-V., and writing-original draft preparation, G.L.S.-O.; writing-review and editing, R.G.-V., L.M.S.-S., and F.A.T.-B.; supervision, R.G.-V., L.M.S.-S., and F.A.T.-B.; funding acquisition, L.M.S.-S. and F.A.T.-B. All authors have read and agreed to the published version of the manuscript.

Funding: This research was funded by HERO Group under the FOODPRINT project: Understanding and reframing minimal processing.

Institutional Review Board Statement: Not applicable.

Informed Consent Statement: Not applicable.

Data Availability Statement: Data sharing is not applicable to this article.

Conflicts of Interest: L.M.S.S. is a member of the Research and Nutrition Department of Hero Group, a Swiss international food manufacturer.

\section{References}

1. Tomás-Barberán, F.A.; Espín, J.C. Effect of Food Structure and Processing on (Poly) phenol—Gut Microbiota Interactions and the Effects on Human Health. Annu. Rev. Food Sci. Technol. 2019, 10, 221-239. [CrossRef] [PubMed]

2. Tomás-Barberán, F.A.; Espín, J.C. Phenolic compounds and related enzymes as determinants of quality in fruits and vegetables. J. Sci. Food Agric. 2001, 81, 853-876. [CrossRef]

3. Nowicka, A.; Kucharska, A.Z.; Sokół-Łẹtowska, A.; Fecka, I. Comparison of polyphenol content and antioxidant capacity of strawberry fruit from 90 cultivars of Fragaria $\times$ ananassa Duch. Food Chem. 2019, 270, 32-46. [CrossRef] [PubMed]

4. Guo, S.; Guan, L.; Cao, Y.; Li, C.; Chen, J.; Li, J.; Liu, G.; Li, S.; Wu, B. Diversity of polyphenols in the peel of apple (Malus sp.) germplasm from different countries of origin. Int. J. Food Sci. Technol. 2016, 51, 222-230. [CrossRef]

5. Aaby, K.; Skrede, G.; Wrolstad, R.E. Phenolic Composition and Antioxidant Activities in Flesh and Achenes of Strawberries (Fragaria ananassa). J. Agric. Food Chem. 2005, 53, 4032-4040. [CrossRef]

6. Buendía, B.; Gil, M.I.; Tudela, J.A.; Gady, A.L.; Medina, J.J.; Soria, C.; López, J.M.; Tomás-Barberán, F.A. HPLC-MS analysis of proanthocyanidin oligomers and other phenolics in 15 strawberry cultivars. J. Agric. Food Chem. 2010, 58, 3916-3926. [CrossRef] 
7. Le Bourvellec, C.; Bouzerzour, K.; Ginies, C.; Renard, C.M.G.C.; Regis, S.; Ple, Y. Phenolic and polysaccharidic composition of applesauce is close to that of apple flesh. J. Food Compos. Anal. 2011, 24, 537-547. [CrossRef]

8. Xu, Y.; Fan, M.; Ran, J.; Zhang, T.; Sun, H.; Dong, M.; Zhang, Z.; Zheng, H. Variation in phenolic compounds and antioxidant activity in apple seeds of seven cultivars. Saudi J. Biol. Sci. 2016, 23, 379-388. [CrossRef]

9. Jakobek, L.; García-Villalba, R.; Tomás-Barberán, F.A. Polyphenolic characterisation of old local apple varieties from Southeastern European region. J. Food Compos. Anal. 2013, 31, 199-211. [CrossRef]

10. Akagić, A.; Vranac, A.; Gaši, F.; Drkenda, P.; Spaho, N.; Žuljević, O.; Kurtović, M.; Musić, O.; Murtić, S.; Hudina, M. Sugars, acids and polyphenols profile of commercial and traditional apple cultivars for processing. Acta Agric. Slov. 2019, 113, 239-250. [CrossRef]

11. Łata, B.; Trampczynska, A.; Paczesna, J. Cultivar variation in apple peel and whole fruit phenolic composition. Sci. Hortic. (Amsterdam) 2009, 121, 176-181. [CrossRef]

12. Fernández-Jalao, I.; Sánchez-Moreno, C.; De Ancos, B. Effect of high-pressure processing on flavonoids, hydroxycinnamic acids, dihydrochalcones and antioxidant activity of apple 'Golden Delicious' from different geographical origin. Innov. Food Sci. Emerg. Technol. 2019, 51, 20-31. [CrossRef]

13. Masi, E.; Taiti, C.; Vignolini, P.; William, A.; Giordani, E.; Heimler, D.; Romani, A.; Mancuso, S. Polyphenols and aromatic volatile compounds in biodynamic and conventional 'Golden Delicious' apples (Malus domestica Bork). Eur. Food Res. Technol. 2017, 243, 1519-1531. [CrossRef]

14. Román, S.; Sánchez-Siles, L.M.; Siegrist, M. The importance of food naturalness for consumers: Results of a systematic review. Trends Food Sci. Technol. 2017, 67, 44-57. [CrossRef]

15. Sanchez-Siles, L.M.; Michel, F.; Román, S.; Bernal, M.J.; Philipsen, B.; Haro, J.F.; Bodenstab, S.; Siegrist, M. The Food Naturalness Index (FNI): An integrative tool to measure the degree of food naturalness. Trends Food Sci. Technol. 2019, 91, 681-690. [CrossRef]

16. Debelo, H.; Li, M.; Ferruzzi, M.G. Processing influences on food polyphenol profiles and biological activity. Curr. Opin. Food Sci. 2020, 32, 90-102. [CrossRef]

17. Pérez-Andrés, J.M.; Charoux, C.M.G.; Cullen, P.J.; Tiwari, B.K. Chemical Modifications of Lipids and Proteins by Nonthermal Food Processing Technologies. J. Agric. Food Chem. 2018, 66, 5041-5054. [CrossRef]

18. Misra, N.N.; Koubaa, M.; Roohinejad, S.; Juliano, P.; Alpas, H.; Inácio, R.S.; Saraiva, J.A.; Barba, F.J. Landmarks in the historical development of twenty fi rst century food processing technologies. Food Res. Int. 2017, 97, 318-339. [CrossRef]

19. Koszucka, A.; Nowak, A. Thermal processing food-related toxicants: A review. Crit. Rev. Food Sci. Nutr. 2019, 59, 3579-3596. [CrossRef]

20. Van Boekel, M.; Fogliano, V.; Pellegrini, N.; Stanton, C.; Scholz, G.; Lalljie, S.; Somoza, V.; Knorr, D.; Jasti, P.R.; Eisenbrand, G. A review on the beneficial aspects of food processing. Mol. Nutr. Food Res. 2010, 54, 1215-1247. [CrossRef]

21. Fam, S.N. High-Pressure Processing in Food. Biointerface Res. Apllied Chem. 2020, 11, 11553-11561.

22. Wael, E.; Johari, E.; Yus, Y.; Rosnah, S.; Anvarjon, A. High Pressure Processing Technology and Equipment Evolution: A Review. J. Eng. Sci. Technol. Rev. 2015, 8, 75-83.

23. Martinez-Monteagudo, S.I.; Saldan, M.D.A. Chemical Reactions in Food Systems at High Hydrostatic Pressure. Food Eng. Rev. 2014, 6, 105-127. [CrossRef]

24. Makita, T. Application of high pressure and thermophysical properties of water to biotechnology. Fluid Phase Equilib. 1992, 76, 87-95. [CrossRef]

25. Tinello, F.; Lante, A. Recent advances in controlling polyphenol oxidase activity of fruit and vegetable products. Innov. Food Sci. Emerg. Technol. 2018, 50, 73-83. [CrossRef]

26. Aaby, K.; Grimsbo, I.H.; Hovda, M.B.; Rode, T.M. Effect of high pressure and thermal processing on shelf life and quality of strawberry purée and juice. Food Chem. 2018, 260, 115-123. [CrossRef]

27. Benito-Román, O.; Sanz, M.T.; Illera, A.E.; Melgosa, R.; Beltrán, S. Polyphenol oxidase (PPO) and pectin methylesterase (PME) inactivation by high pressure carbon dioxide (HPCD) and its applicability to liquid and solid natural products. Catal. Today. 2019, 346, 112-120. [CrossRef]

28. Smeriglio, A.; Barreca, D.; Bellocco, E.; Trombetta, D. Proanthocyanidins and hydrolysable tannins: Occurrence, dietary intake and pharmacological effects. Br. J. Pharmacol. 2017, 174, 1244-1262. [CrossRef]

29. Vrhovsek, U.; Rigo, A.; Tonon, D.; Mattivi, F. Quantitation of polyphenols in different apple varieties. J. Agric. Food Chem. 2004, 52, 6532-6538. [CrossRef]

30. Rauf, A.; Imran, M.; Abu-Izneid, T.; Iahtisham-Ul-Haq; Patel, S.; Pan, X.; Naz, S.; Sanches Silva, A.; Saeed, F.; Rasul Suleria, H.A. Proanthocyanidins: A comprehensive review. Biomed. Pharmacother. 2019, 116. [CrossRef]

31. Oliveira, A.; Coelho, M.; Alexandre, E.M.C.; Almeida, D.P.F.; Pintado, M. Long-Term Frozen Storage and Pasteurization Effects on Strawberry Polyphenols Content. Food Bioprocess Technol. 2015, 8, 1838-1844. [CrossRef]

32. Oliveira, A.; Almeida, D.P.F.; Pintado, M. Changes in Phenolic Compounds During Storage of Pasteurized Strawberry. Food Bioprocess Technol. 2014, 7, 1840-1846. [CrossRef]

33. Garzoli, S.; Cairone, F.; Carradori, S.; Mocan, A.; Menghini, L.; Paolicelli, P.; Ak, G.; Zengin, G.; Cesa, S. Effects of Processing on Polyphenolic and Volatile Composition and Fruit Quality of Clery Strawberries. Antioxidants 2020, 9, 632. [CrossRef] [PubMed] 
34. Álvarez-Fernández, M.A.; Hornedo-Ortega, R.; Cerezo, A.B.; Troncoso, A.M.; García-Parrilla, M.C. Effects of the strawberry (fragaria ananassa) purée elaboration process on non-anthocyanin phenolic composition and antioxidant activity. Food Chem. 2014, 164, 104-112. [CrossRef]

35. Howard, L.R.; Brownmiller, C.; Prior, R.L. Improved color and anthocyanin retention in strawberry puree by oxygen exclusion. J. Berry Res. 2014, 4, 107-116. [CrossRef]

36. Holzwarth, M.; Korhummel, S.; Kammerer, D.R.; Carle, R. Thermal inactivation of strawberry polyphenoloxidase and its impact on anthocyanin and color retention in strawberry (Fragaria x ananassa Duch) pure. Eur. Food Res. Technol. 2012, 235, 1171-1180. [CrossRef]

37. Teleszko, M.; Nowicka, P.; Wojdyło, A. Effect of cultivar and storage temperature on identification and stability of polyphenols in strawberry cloudy juices. J. Food Compos. Anal. 2016, 54, 10-19. [CrossRef]

38. Bodelón, O.G.; Avizcuri, J.; Dizy, M. Pressurization and cold storage of strawberry purée: Colour, anthocyanins, ascorbic acid and pectin methylesterase. LWT Food Sci. Technol. 2013, 52, 123-130. [CrossRef]

39. Marszałek, K.; Woźniak, L.; Skapska, S. The application of high pressure-mild temperature processing for prolonging the shelf-life of strawberry purée. High Press. Res. 2016, 36, 220-234. [CrossRef]

40. Stübler, A.S.; Lesmes, U.; Juadjur, A.; Heinz, V.; Rauh, C.; Shpigelman, A.; Aganovic, K. Impact of pilot-scale processing (thermal, PEF, HPP) on the stability and bioaccessibility of polyphenols and proteins in mixed protein- and polyphenol-rich juice systems. Innov. Food Sci. Emerg. Technol. 2020, 64, 102426. [CrossRef]

41. Marszałek, K.; Mitek, M.; Skapska, S. The effect of thermal pasteurization and high pressure processing at cold and mild temperatures on the chemical composition, microbial and enzyme activity in strawberry purée. Innov. Food Sci. Emerg. Technol. 2015, 27, 48-56. [CrossRef]

42. Terefe, N.S.; Kleintschek, T.; Gamage, T.; Fanning, K.J.; Netzel, G.; Versteeg, C.; Netzel, M. Comparative effects of thermal and high pressure processing on phenolic phytochemicals in different strawberry cultivars. Innov. Food Sci. Emerg. Technol. 2013, 19, 57-65. [CrossRef]

43. Marszałek, K.; Woźniak, Ł.; Skapska, S.; Mitek, M. High pressure processing and thermal pasteurization of strawberry purée: Quality parameters and shelf life evaluation during cold storage. J. Food Sci. Technol. 2017, 54, 832-841. [CrossRef]

44. Cao, X.; Zhang, Y.; Zhang, F.; Wang, Y.; Yi, J.; Liao, X. Effects of high hydrostatic pressure on enzymes, phenolic compounds, anthocyanins, polymeric color and color of strawberry pulps. J. Sci. Food Agric. 2011, 91, 877-885. [CrossRef]

45. Cao, X.; Bi, X.; Huang, W.; Wu, J.; Hu, X.; Liao, X. Changes of quality of high hydrostatic pressure processed cloudy and clear strawberry juices during storage. Innov. Food Sci. Emerg. Technol. 2012, 16, 181-190. [CrossRef]

46. Kim, A.N.; Lee, K.Y.; Rahman, M.S.; Kim, H.J.; Kerr, W.L.; Choi, S.G. Thermal treatment of apple puree under oxygen-free condition: Effect on phenolic compounds, ascorbic acid, antioxidant activities, color, and enzyme activities. Food Biosci. 2021, 39, 100802. [CrossRef]

47. Murtaza, A.; Iqbal, A.; Marszałek, K.; Iqbal, M.A.; Ali, S.W.; Xu, X.; Pan, S.; Hu, W. Enzymatic, phyto-, and physicochemical evaluation of apple juice under high-pressure carbon dioxide and thermal processing. Foods 2020, 9, 243. [CrossRef]

48. Alongi, M.; Verardo, G.; Gorassini, A.; Anese, M. Effect of pasteurization on in vitro $\alpha$-glucosidase inhibitory activity of apple juice. LWT Food Sci. Technol. 2018, 98, 366-371. [CrossRef]

49. Knebel, T.; Braun, P.; Dietrich, H. Degradation kinetics of anthocyanins and polyphenols during storage of red apple juice produced from red-fleshed apples. Eur. Food Res. Technol. 2018, 244, 1741-1750. [CrossRef]

50. Tian, Y.; Sun, L.; Yang, Y.; Gou, X.; Niu, P.; Guo, Y. Changes in the physicochemical properties, aromas and polyphenols of not from concentrate (NFC) apple juice during production. CyTA J. Food 2018, 16, 755-764. [CrossRef]

51. De Paepe, D.; Valkenborg, D.; Coudijzer, K.; Noten, B.; Servaes, K.; De Loose, M.; Voorspoels, S.; Diels, L.; Van Droogenbroeck, B. Thermal degradation of cloudy apple juice phenolic constituents. Food Chem. 2014, 162, 176-185. [CrossRef]

52. Szczepańska, J.; Pinto, C.A.; Skapska, S.; Saraiva, J.A.; Marszałek, K. Effect of static and multi-pulsed high pressure processing on the rheological properties, microbial and physicochemical quality, and antioxidant potential of apple juice during refrigerated storage. LWT Food Sci. Technol. 2021, 150, 112038. [CrossRef]

53. Fernández-Jalao, I.; Balderas, C.; Sánchez-Moreno, C.; De Ancos, B. Impact of an in vitro dynamic gastrointestinal digestion on phenolic compounds and antioxidant capacity of apple treated by high-pressure processing. Innov. Food Sci. Emerg. Technol. 2020, 66, 102486. [CrossRef]

54. Marszalek, K.; Szczepańska, J.; Starzonek, S.; Wozniak, L.; Trych, U.; Skapska, S.; Rzoska, S.; Saraiva, J.A.; Lorenzo, J.M.; Barba, F.J. Enzyme inactivation and evaluation of physicochemical properties, sugar and phenolic profile changes in cloudy apple juices after high pressure processing, and subsequent refrigerated storage. Food Process. Eng. 2019, 42, e13034. [CrossRef]

55. Castañeda-Ovando, A.; Pacheco-Hernández, M.d.L.; Páez-Hernández, M.E.; Rodríguez, J.A.; Galán-Vidal, C.A. Chemical studies of anthocyanins: A review. Food Chem. 2009, 113, 859-871. [CrossRef]

56. Krga, I.; Milenkovic, D. Anthocyanins: From Sources and Bioavailability to Cardiovascular-Health Benefits and Molecular Mechanisms of Action. J. Agric. Food Chem. 2019, 67, 1771-1783. [CrossRef]

57. Bueno, J.M.; Sáez-Plaza, P.; Ramos-Escudero, F.; Jiménez, A.M.; Fett, R.; Asuero, A.G. Analysis and Antioxidant Capacity of Anthocyanin Pigments. Part II: Chemical Structure, Color, and Intake of Anthocyanins. Crit. Rev. Anal. Chem. 2012, 42, 126-151. [CrossRef] 
58. Tena, N.; Martín, J.; Asuero, A.G. State of the art of anthocyanins: Antioxidant activity, sources, bioavailability, and therapeutic effect in human health. Antioxidants 2020, 9, 451. [CrossRef]

59. Mattioli, R.; Francioso, A.; Mosca, L.; Silva, P. Anthocyanins: A Comprehensive Review of Their Chemical Properties and Health Effects on Cardiovascular and Neurodegenerative Diseases. Molecules 2020, 25, 3809. [CrossRef]

60. Motilva, M.; Bars-cortina, D.; Sakhawat, A.; Pi, C. Chemopreventive effects of anthocyanins on colorectal and breast cancer: A review. Semin. Cancer Biol. 2021, 6, 1203. [CrossRef]

61. Li, D.; Wang, P.; Luo, Y.; Zhao, M.; Chen, F. Health benefits of anthocyanins and molecular mechanisms: Update from recent decade. Crit. Rev. Food Sci. Nutr. 2017, 57, 1729-1741. [CrossRef] [PubMed]

62. Skrede, G.; Wrolstad, R.E.; Lea, P.; Enersen, G. Color Stability of Strawberry and Blackcurrant Syrups. J. Food Sci. $1992,57$. [CrossRef]

63. Nikkhah, E.; Khaiamy, M.; Heidary, R.; Azar, A.S. The effect of ascorbic acid and $\mathrm{H} 2 \mathrm{O} 2$ treatment on the stability of anthocyanin pigments in berries. Turk J. Biol. 2010, 34, 47-53. [CrossRef]

64. Pacheco-Palencia, L.A.; Hawken, P.; Talcott, S.T. Phytochemical, antioxidant and pigment stability of ac (Euterpe oleracea Mart) as affected by clarification, ascorbic acid fortification and storage. Food Res. Int. 2007, 40, 620-628. [CrossRef]

65. Bakkalbasi, E.; Mentes, O.; Artik, N. Food ellagitannins-occurrence, effects of processing and storage. Crit. Rev. Food Sci. Nutr. 2009, 49, 283-298. [CrossRef]

66. Williner, M.R.; Pirovani, M.E.; Güemes, D.R. Ellagic acid content in strawberries of different cultivars and ripening stages. J. Sci. Food Agric. 2003, 83, 842-845. [CrossRef]

67. Tomás-Barberán, F.A.; González-Sarrías, A.; García-Villalba, R.; Núñez-Sánchez, M.A.; Selma, M.V.; García-Conesa, M.T.; Espín, J.C. Urolithins, the rescue of "old" metabolites to understand a "new" concept: Metabotypes as a nexus among phenolic metabolism, microbiota dysbiosis, and host health status. Mol. Nutr. Food Res. 2017, 61. [CrossRef]

68. Cerdá, B.; Periago, P.; Espín, J.C.; Tomás-Barberán, F.A. Identification of urolithin A as a metabolite produced by human colon microflora from ellagic acid and related compounds. J. Agric. Food Chem. 2005, 53, 5571-5576. [CrossRef]

69. Al-Harbi, S.A.; Abdulrahman, A.O.; Zamzami, M.A.; Khan, M.I. Urolithins: The Gut Based Polyphenol Metabolites of Ellagitannins in Cancer Prevention, a Review. Front. Nutr. 2021, 8, 1-15. [CrossRef]

70. Okuda, T.; Yoshida, T.; Hatano, T. Ellagitannins as active constituents of medicinal plants. Planta Med. 1989, 55, 117-122. [CrossRef]

71. Truchado, P.; Larrosa, M.; García-Conesa, M.T.; Cerdá, B.; Vidal-Guevara, M.L.; Tomás-Barberán, F.A.; Espín, J.C. Strawberry processing does not affect the production and urinary excretion of urolithins, ellagic acid metabolites, in humans. J. Agric. Food Chem. 2012, 60, 5749-5754. [CrossRef]

72. Landete, J.M. Ellagitannins, ellagic acid and their derived metabolites: A review about source, metabolism, functions and health. Food Res. Int. 2011, 44, 1150-1160. [CrossRef]

73. Materska, M. Quercetin and its derivatives: Chemical structure and bioactivity-A review. Polish J. Food Nutr. Sci. 2008, 58, 407-413.

74. Kamiloglu, S. Effect of different freezing methods on the bioaccessibility of strawberry polyphenols. Int. J. Food Sci. Technol. 2019, 54, 2652-2660. [CrossRef]

75. Herrmann, K. Flavonols and flavones in food plants: A review. Int. J. Food Sci. Technol. 1976, 11, 433-448. [CrossRef]

76. Lesjak, M.; Beara, I.; Pinta, D.; Majki, T.; Bekvalac, K.; Or, D.; Mimica-duki, N. Antioxidant and anti-in fl ammatory activities of quercetin and its derivatives. J. Funct. Foods 2018, 40, 68-75. [CrossRef]

77. Panthi, V.K.; Kaushal, S.; Adhikari, B.; Basnet, N.; Chaudary, D.; Parajuli, R.; Pokhrel, P. A Review of Quercetin: Anticancer Activity. Int. J. Innov. Res. Rev. 2020, 4, 1-7.

78. Islam, S.; Quispe, C.; Hossain, R.; Islam, M.T. Neuropharmacological Effects of Quercetin: A Literature-Based Review. Front. Pharmacol. 2021, 12, 1-16. [CrossRef]

79. Alongi, M.; Verardo, G.; Gorassini, A.; Lemos, M.A.; Hungerford, G.; Cortella, G.; Anese, M. Phenolic content and potential bioactivity of apple juice as affected by thermal and ultrasound pasteurization. Food Funct. 2019, 10, 7366-7377. [CrossRef]

80. Tomás-Barberán, F.; Garcia-viguera, C.; Nieto, J.L.; Ferreres, F. Dihydrochalcones from apple juices and jams. Food Chem. 1993, 46, 33-36. [CrossRef]

81. Tian, L.; Cao, J.; Zhao, T.; Liu, Y.; Khan, A.; Cheng, G. The bioavailability, extraction, biosynthesis and distribution of natural dihydrochalcone: Phloridzin. Int. J. Mol. Sci. 2021, 22, 962. [CrossRef]

82. Viet, M.H.; Chen, C.Y.; Hu, C.K.; Chen, Y.R.; Li, M.S. Discovery of dihydrochalcone as potential lead for Alzheimer's disease: In Silico and in Vitro study. PLoS ONE 2013, 8, e79151. [CrossRef]

83. Raphaelli, C.d.O.; Azevedo, J.G.; Pereira, E.d.S.; Vinholes, J.R.; Camargo, T.M.; Hoffmann, J.F.; Ribeiro, J.A.; Vizzotto, M.; Rombaldi, C.V.; Wink, M.R.; et al. Phenolic-rich apple extracts have photoprotective and anti-cancer effect in dermal cells. Phytomedicine Plus 2021, 1, 100112. [CrossRef]

84. Tomás-Barberán, F.; Clifford, M. Flavanones, chalcones and dihydrochalcones—Nature, occurrence and dietary burden. J. Sci. Food Agric. 2000, 80, 1073-1080. [CrossRef]

85. Oszmianski, J.; Wolniak, M.; Wojdylo, A.; Wawer, I. Influence of apple puree preparation and storage on polyphenol contents and antioxidant activity. Food Chem. 2008, 107, 1473-1484. [CrossRef] 
86. Teixeira, J.; Gaspar, A.; Garrido, E.M.; Garrido, J.; Borges, F. Hydroxycinnamic Acid Antioxidants: An Electrochemical Overview. Biomed. Res. Int. 2013, 2013, 1-11. [CrossRef]

87. Sova, M. Activities and Health Benefits of Hydroxycinnamic Acids and Their Metabolites. Nutrients 2020, 12, 2190. [CrossRef]

88. Vinholes, J.; Silva, B.M.; Silva, L.R. Hydroxycinnamic acids (HCAS): Structure, Biological properties and health effects. Adv. Med. Biol. 2019, 88, 1-33.

89. Tsao, R.; Yang, R.; Young, J.C.; Zhu, H. Polyphenolic profiles in eight apple cultivars using high-performance liquid chromatography (HPLC). J. Agric. Food Chem. 2003, 51, 6347-6353. [CrossRef]

90. Coman, V.; Vodnar, D.C. Hydroxycinnamic acids and human health: Recent advances. Soc. Chem. Ind. 2019. [CrossRef]

91. Rocha, L.D.; Monteiro, M.C.; Teodoro, A.J. Anticancer Properties of Hydroxycinnamic Acids -A Review. Cancer Clin. Oncol. 2012, 1, 109-121. [CrossRef] 